\title{
1 Trehalose-6-phosphate links singlet oxygen-induced signalling 2 with metabolic signalling in Chlamydomonas reinhardtii
}

4 Waeil Al Youssef ${ }^{1}$, Regina Feil ${ }^{2}$, Maureen Saint-Sorny ${ }^{3}$, Xenie Johnson ${ }^{3}$, John E. Lunn ${ }^{2}$, 5 Bernhard Grimm ${ }^{1}$, and Pawel Brzezowski ${ }^{*}$

\section{Affiliation:}

$8{ }^{1}$ Humboldt-Universität zu Berlin, Institut für Biologie/Pflanzenphysiologie, 10115 Berlin, 9 Germany

$10{ }^{2}$ Max Planck Institute of Molecular Plant Physiology, 14476 Potsdam-Golm, Germany

$11{ }^{3}$ Aix Marseille Université, CNRS, CEA, Institut de Biosciences et Biotechnologies AixMarseille, Photosynthesis and Environment team, CEA Cadarache, 13108 Saint-Paul-lezDurance, France.

*e-mail: pawel.brzezowski@hu-berlin.de

\section{Abstract}

Singlet oxygen $\left({ }^{1} \mathrm{O}_{2}\right)$ induces retrograde signalling in chloroplasts. Using a novel mutant screen, we identified a mutation in the TREHALOSE-6-PHOSPHATE PHOSPHATASE 1 (T6PP1) gene that results in accumulation of trehalose 6-phosphate, a reprogramming of cell metabolism, and impairment of ${ }^{1} \mathrm{O}_{2}$-induced retrograde signalling in Chlamydomonas reinhardtii. From transcriptomic analysis and metabolite profiling, we conclude that accumulation or deficiency of certain metabolites directly affect ${ }^{1} \mathrm{O}_{2}$-signalling. ${ }^{1} \mathrm{O}_{2}$-inducible GLUTATHIONE PEROXIDASE 5 (GPX5) gene expression is suppressed by increased content of fumarate, an intermediate in the tricarboxylic acid cycle (TCA cycle) in mitochondria and dicarboxylate metabolism in the cytosol, while it is promoted by another TCA cycle intermediate, aconitate. Furthermore, genes encoding known essential components of chloroplast-to-nucleus ${ }^{1} \mathrm{O}_{2}$-signalling show decreased transcript levels in a t6ppl mutant, which can be rescued by exogenous application of aconitate. We demonstrate that chloroplast retrograde signalling involving ${ }^{1} \mathrm{O}_{2}$ depends on mitochondrial and cytosolic processes and that the metabolic status of the cell determines the response to ${ }^{1} \mathrm{O}_{2}$. 


\section{Introduction}

Nearly forty years ago, tetrapyrrole biosynthesis (TBS) intermediates were proposed to be involved in chloroplast retrograde (organelle-to-nucleus) signalling. This conclusion was based on the observation that accumulation of chlorophyll precursors negatively affects transcript level of light-harvesting chlorophyll a/b-binding (LHCB) protein ${ }^{1}$. Later studies on chloroplast retrograde signalling involved mutants ${ }^{2}$, treatment with a carotenoid biosynthesis inhibitor, norflurazon ${ }^{3}$, or TBS inhibitors, such as $\alpha, \alpha$-dipyridyl ${ }^{1}$ or thujaplicin ${ }^{4}$. Experiments with norflurazon led to the discovery of the genomes uncoupled (gun) mutants with a common phenotype of chloroplast status-independent expression of photosynthesisassociated nuclear genes ${ }^{3}$.

It is noteworthy that treatment with inhibitors causing carotenoid deficiencies results in generation of reactive oxygen species (ROS) and photooxidative damage to chloroplasts. Furthermore, the end-products of TBS, haem and chlorophyll, as well as many of their intermediates (Supplementary Fig. 1) are light-absorbing and redox-reactive molecules, capable to generate ROS. Singlet oxygen $\left({ }^{1} \mathrm{O}_{2}\right)$ can be produced through interaction of ground (triplet)-state oxygen $\left({ }^{3} \mathrm{O}_{2}\right)$ with triplet-state chlorophyll or TBS intermediates, e.g. protoporphyrin IX (Proto), which are excited by light ${ }^{5}$. ROS are also metabolic products of other cellular processes in plants, such as photosynthesis and respiration.

Although ${ }^{1} \mathrm{O}_{2}$ is not considered to be the most reactive oxygen species, it is thought to be the major ROS involved in photooxidative damage ${ }^{6}$. However, it was shown that production of ${ }^{1} \mathrm{O}_{2}$ in the chloroplast induces stress responses that do not result exclusively from physicochemical damage, but also rely on signal transduction triggered by $\mathrm{ROS}^{7}$. Additionally, studies on the fluorescent (flu) mutant of Arabidopsis thaliana, which is impaired in regulation of TBS and accumulates protochlorophyllide (Supplementary Fig. 1), demonstrated that ${ }^{1} \mathrm{O}_{2}$-retrograde signalling is integrated into a broader signalling network and affects not only stress responses but also metabolism ${ }^{8}$.

However, ${ }^{1} \mathrm{O}_{2}$ has a short half-life (about $200 \mathrm{~ns}$ ) in the cell ${ }^{9}$. As a result, the distance that it may move was calculated to be approximately $10 \mathrm{~nm}$, based on predicted diffusion rates $^{10,11}$. Its diffusion range is also limited due to its high reactivity with membrane lipids. Therefore, ${ }^{1} \mathrm{O}_{2}$ could play a specific role as an activator of a stress response only if it is detected close to its source, which strongly suggests that other components mediating the ${ }^{1} \mathrm{O}_{2}$ signals should exist. Alternatively, altered metabolite contents triggered by ${ }^{1} \mathrm{O}_{2}$ may also mediate ${ }^{1} \mathrm{O}_{2}$-retrograde signalling, or as proposed by Pfannschmidt ${ }^{12}$, a certain metabolic signature may be required to trigger changes in nuclear gene expression. 
Despite the profound effect of ${ }^{1} \mathrm{O}_{2}$ on the chloroplast redox state and its apparent involvement in altering nuclear gene expression, little is known about the components involved in ${ }^{1} \mathrm{O}_{2}$-dependent retrograde signalling. The protein factors identified so far include EXECUTER1 (EX1) and EX2 in the flu mutant of $A$. thaliana $^{13}$, PSBP DOMAINCONTAINING PROTEIN 2 (PSBP2 ${ }^{14}$ and SINGLET OXYGEN ACCLIMATION (SAK1 ${ }^{15}$ in Chlamydomonas reinhardtii, or METHYLENE BLUE SENSITIVITY (MBS1 ${ }^{16}$ shown to be involved in ${ }^{1} \mathrm{O}_{2}$-signalling in both $A$. thaliana and C. reinhardtii.

In our search for tetrapyrrole-signalling components, we hypothesized that ${ }^{1} \mathrm{O}_{2}$ generated via the photosensitizing activity of Proto, rather than porphyrin accumulation itself, triggers signalling cascades that alter nuclear gene expression. Therefore, we used a $C$. reinhardtii mutant $\operatorname{chlD}-1^{17}$ that accumulates Proto due to a dysfunctional Mg-chelatase (MgCh, Supplementary Fig.1). The GENOMES UNCOUPLED 4 (GUN4) protein involved in $\mathrm{MgCh}$ function (Supplementary Fig. 1) and signalling degrades upon Proto accumulation $^{18}$. However, chlD-1 with introduced over-expression of GUN4, chlD-1/GUN4, showed higher GUN4 content than chlD-1, but retained the chlorophyll-free phenotype ${ }^{18}$. Additionally, chlD-1/GUN4 demonstrated higher expression of GPX5 and higher tolerance to ${ }^{1} \mathrm{O}_{2}$ than $\operatorname{chlD}-1^{18}$. Thus, to minimize the GUN4-deficient phenotype and to maintain high and stable ${ }^{1} \mathrm{O}_{2}$-inducibility of GPX5 expression, chlD-1/GUN4 was used as a background strain instead of chlD-1 in the present study to monitor ${ }^{1} \mathrm{O}_{2}$-signalling. For this purpose, expression of the GLUTATHIONE PEROXIDASE 5 (GPX5) gene, which is specifically induced by ${ }^{1} \mathrm{O}_{2}$ in $C$. reinhardtii ${ }^{19,20}$ was used as a marker gene. The ${ }^{1} \mathrm{O}_{2}$-inducible promoter region of GPX5 fused to the promoterless ARYLSULFATASE 2 gene (ARS2) was shown to be an effective reporter to study $G P X 5$ expression $^{14,20,21}$.

Mutagenesis of the strain carrying the GPX5-ARS2 reporter (in a chlD-1/GUN4 mutant background), followed by a screening for decreased reporter gene expression identified a mutant impaired in ${ }^{1} \mathrm{O}_{2}$-signalling. The lack of the retrograde response to ${ }^{1} \mathrm{O}_{2}$ in this mutant was verified to decrease expression of $G P X 5$, as well as $S A K 1, M B S$, and $P S B P 2$. The causal mutation was found in a gene annotated as TREHALOSE 6-PHOSPHATE PHOSPHATASE (hereafter T6PP1). Besides clear impairment in ${ }^{1} \mathrm{O}_{2}$-signalling, mutation in T6PP1 also resulted in accumulation of trehalose 6-phosphate (Tre6P). Therefore, T6PP1 is the first confirmed phosphatase acting on Tre6P in C. reinhardtii and in fact the first enzyme with a confirmed function in trehalose metabolism in this organism. Our data indicate that accumulation of Tre6P causes changes to the expression of genes involved in several metabolic pathways. However, Tre6P is an intermediate rather than the primary cause of the 
103 impaired ${ }^{1} \mathrm{O}_{2}$-signalling. Here using a combination of genetics, gene expression and

104 metabolic phenotypes, we reveal a complex interaction between chloroplast, mitochondria 105 and cytosol in ${ }^{1} \mathrm{O}_{2}$ signal transmission to the nucleus.

\section{Results}

108 Forward genetics screen to identify components of the ${ }^{1} \mathbf{O}_{\mathbf{2}}$-signalling

109 To generate a mutant impaired in ${ }^{1} \mathrm{O}_{2}$-dependent signalling, we first created a reporter strain 110 in a known ${ }^{1} \mathrm{O}_{2}$-generating mutant, which was subsequently subjected to mutagenesis. The 111 GPX5-ARS2 reporter construct (Supplementary Fig. 2) was introduced into the genome of 112 chlD-1/GUN4 to express the ARS2 protein in a ${ }^{1} \mathrm{O}_{2}$-dependent manner, and the transformants 113 were tested for the enzymatic activity of ARS2. The reporter strain, which showed the lowest 114 ARS2 activity in darkness and the highest activity in light was named signalling Reporter 115 (sigRep; Fig. 1a) and was used in further applications. Expression kinetics of the cytosolic $116\left(G P X 5_{c y t}\right)$ and chloroplastic $\left(G P X 5_{c p}\right)$ version of $G P X 5^{22}$; Supplementary Fig. 2), as well as 117 the GPX5-ARS2 construct were examined by quantitative Real-Time PCR (qRT-PCR) upon 118 transfer from dark to light. As expected, higher induction of $G P X 5_{c p}$ and $G P X 5_{c y t}$ were 119 observed upon light illumination in sigRep compared to WT (Fig. 1b). Induced GPX5-ARS2 120 expression in sigRep in the light (Fig. 1c) confirmed the results obtained in the ARS activity assay (Fig. 1a).

Subsequently, a random mutagenesis was performed on sigRep using bleomycin resistance cassette $\left(b^{\mathrm{R}}{ }^{\mathrm{R}}\right.$ ) as an insert, followed by screening to isolate strains with lower or undetectable ARS2 activity in the light compared to sigRep, and thus, possibly disrupted ${ }^{1} \mathrm{O}_{2}$ dependent signalling. Screen of 804 transformants allowed us to isolate nine, which were named genomes uncoupled $\underline{\text { Singlet }}$ Oxygen Signalling (gunSOS). The gunSOS1 mutant (Fig. 1d) was selected for further analysis. Higher sensitivity to light was recorded in gunSOS1 compared to the sigRep background strain (Fig. 1e), in agreement with a higher sensitivity or impaired acclimation to ${ }^{1} \mathrm{O}_{2}$. Mutant impaired in ${ }^{1} \mathrm{O}_{2}$-signalling showed similar Proto accumulation in light compared to sigRep and chlD-1/GUN4 (Supplementary Fig. 3), which eliminated the possibility that spurious mutations in gunSOS1 resulted in reduced content of this photosensitizer.

The ble ${ }^{\mathrm{R}}$ insertion in gunSOS1 was located in the first exon of the gene annotated as TREHALOSE 6-PHOSPHATE PHOSPHATASE 1 (here T6PP1; locus Cre12.g497750) in the JGI portal (Department of Energy Joint Genome Institute, https://phytozomenext.jgi.doe.gov, v13, genome v5.6; Fig. 1f). The T6PPl transcript abundance was 
137 determined in gunSOS1 and compared to sigRep by qRT-PCR using primers annealing to the

138 coding sequence upstream of the insertion site. At $2 \mathrm{~h}$ after transfer from dark to light, T6PP 1 139 mRNA content increased 2.5-fold in sigRep compared to WT, while it was virtually absent in 140 gunSOS1 (Fig. 1g). Expression of GPX5 $5_{c y t}$ and $G P X 5_{c p}$ was nearly 10 and 8 times lower in 141 gunSOS1 compared to sigRep, respectively (Fig. 1h). The transcript abundance for GPX5142 ARS2 was 7 times lower in gunSOS1 than in sigRep (Fig. 1i), and explains undetectable 143 ARS2 activity in the ${ }^{1} \mathrm{O}_{2}$-signalling mutant (Fig. 1d).

\section{C. reinhardtii T6PP1 is a functional Tre6P phosphatase}

146 While gunSOS1 is clearly impaired in ${ }^{1} \mathrm{O}_{2}$-signalling, it was necessary to determine the primary phenotype caused by the mutation in T6PP1. The content of Tre6P in sigRep and gunSOS1 was determined by anion-exchange high performance liquid chromatography coupled to tandem mass spectrometry (LC-MS/MS). Tre6P accumulation increased in gunSOS1 upon exposure to light in a time-dependent manner, while it decreased in sigRep (Fig. 2a). Accumulation of Tre6P in gunSOS1 indicates that $C$. reinhardtii T6PP1 is a functional phosphatase dephosphorylating Tre6P to trehalose. Subsequently, T6PP1 expression was found to be induced in the light in sigRep, but not in the WT, indicating its inducibility by photooxidative stress rather than light (Fig. 2b). Mutation of the T6PP1 gene would be expected to lower Tre6P phosphatase activity and dephosphorylation of Tre6P to trehalose, but we also observed accumulation of trehalose in gunSOS1 (Fig. 2c). This apparent discrepancy can be explained by the existence of another enzyme in $C$. reinhardtii with possible Tre6P phosphatase activity, in combination with higher levels of Tre6P (see Discussion section).

Rescue of the T6PP1 deficiency in gunSOS1 was performed with the isolated genomic DNA fragment carrying the wild-type T6PP1 gene. Several independent transformants 162 showed rescued ${ }^{1} \mathrm{O}_{2}$-signalling, which was indicated by the GPX5-ARS2 expression 163 determined in the ARS assay, and data from three representative strains are shown in Supplementary Fig. 4a. The analysed rescued strains also showed increased $G P X 5_{c p}$ transcript content compared to gunSOS1 (Supplementary Fig. 4b). A peptide-specific antibody for T6PP1 was produced to compare the protein content in gunSOS1 to strains showing ${ }^{1} \mathrm{O}_{2}$ dependent signalling. A faint immune signal of approximately $43 \mathrm{kDa}$ was detected in gunSOS1 and chlD-1, but two narrow bands were seen in chlD-1/GUN4, sigRep and the T6PP1-rescued strains (Fig. 2d). WT and gunSOS1 showed an additional unspecific immunoreaction with protein of approximately $53 \mathrm{kDa}$. As expected, GPX5 content was 
171 lower in gunSOS1 compared to sigRep (Fig. 2d), which correlated with qRT-PCR results on

$172 G P X 5$ transcript levels (Fig. 1 h). Strains with rescued ${ }^{1} \mathrm{O}_{2}$-signalling, T6PP1-R1, -R2, and -

$173 R 3$, showed increased content of GPX5 (Fig. 2d) and higher tolerance to light compared to 174 gunSOS1 (Fig. 2e).

175

\section{The gunSOS1 mutant shows altered metabolism compared to sigRep}

177 In $A$. thaliana and other angiosperms, Tre6P functions as a sucrose signal and homeostatic 178 regulator of sucrose metabolism ${ }^{23,24}$, which links plant growth and development to the 179 availability of sucrose ${ }^{25}$, reviewed $\mathrm{in}^{26}$. To determine the effect of accumulated Tre6P on 180 metabolism in C. reinhardtii, we performed comparative metabolite profiling of gunSOS1 181 and sigRep (Supplementary Table 1), which revealed a relatively wide range of variation. 182 Aside from Tre6P and trehalose, 11 out of 27 analysed metabolites showed significantly 183 increased content in gunSOS1 compared to sigRep in the light (Figure 3a). By means of the 184 MetaboAnalyst 5.0 portal (https://www.metaboanalyst.ca) these metabolites were assigned to 185 the metabolic processes in the cell. A hypergeometric test indicated the most affected 186 metabolic pathways, i.e. the TCA cycle, pyruvate metabolism, and starch and sucrose 187 metabolism (Fig. 3b, Supplementary Table 2). Eight metabolites showed significantly 188 decreased content in gunSOS1 compared to sigRep (Fig. 4a), with the highest impact 189 (MetaboAnalyst 5.0) on fructose and mannose metabolism, amino sugar and nucleotide sugar metabolism, and starch and sucrose metabolism (Fig. 4b, Supplementary Table 3). Metabolites, which did not show a significant change in gunSOS1 compared to sigRep are presented in Supplementary Fig. 5.

Starch content was determined in gunSOS1 and compared to sigRep, because of changes in several intermediates of starch and sucrose metabolism (Fig. 3b and Fig. 4b). Indeed, gunSOS1 accumulated only $60 \%$ of the starch content of sigRep when the strains were grown in acetate-supplemented media (TAP, Fig. 4c). Both sigRep and gunSOS1 are obligate heterotrophs. SigRep contained less starch after transfer from TAP and cultivation in TP for $48 \mathrm{~h}$, whereas the starch content of gunSOS1 was essentially unchanged (Fig. 4c), indicating that there was net degradation of starch in sigRep but not in gunSOS1.Starch formation is strongly induced during nitrogen $(\mathrm{N})$ deprivation ${ }^{27}$. The sigRep cells did not increase starch reserves after 3 days in N-deficient medium, suggesting that starch accumulation had already reached its maximum under non-stress conditions. The starch content in gunSOS1 deprived of $\mathrm{N}$ did increase by $50 \%$ and was similar to the content in sigRep (Fig. 4c). We conclude that sigRep accumulates high levels of starch as a 
205 consequence of the chlD-1/GUN4 genetic background and that the accumulation of Tre6P

206 represses this response in gunSOS1 leading to a lower accumulation of starch. The gunSOS1

207 cells are still capable of responding to the N-deprivation stress and accumulating starch 208 despite disturbed metabolism. Starch degradation on the other hand, is compromised in 209 gunSOS1, which could indicate a general impairment of catabolic processes or a defect in 210 sensing carbon limitation. The map-overview of the selected metabolic pathways with 211 depicted metabolites that showed different content, or were not changed, in gunSOS1 relative 212 to sigRep is presented in Figure 5.

Increased Tre6P content alters metabolism in $C$. reinhardtii via alteration of the transcriptome

216 To determine the extent to which chloroplast retrograde signalling is affected in gunSOS1, we performed comparative RNA-sequencing (RNA-seq) of the transcriptomes of gunSOS1 and sigRep upon transfer from dark to light. The analysis showed that 8120 genes were expressed in both strains, but 1377 and 1087 additional genes were uniquely expressed in gunSOS1 and sigRep, respectively (Fig. 6a, detailed listing can be found in Supplementary Data Set 1). Furthermore, differential gene expression analysis showed that the transcript contents of 1606 genes and 1706 genes were lower or higher, respectively, in gunSOS1 compared to sigRep (Supplementary Data Set 2).

Based on the KEGG ontology (http://www.kegg.jp/) several genes associated with metabolic pathways were down- (Fig. 6b) or up-regulated (Fig. 6c) in gunSOS1 compared to sigRep (Supplementary Data Set 3). Given the strong overlap between differentially expressed genes and corresponding pathway intermediates, we hypothesized that the altered metabolism can be directly explained by altered gene expression. To decipher a complex correlation between altered signalling, gene expression and metabolism, accumulation or deficiency in metabolites from given pathway(s) was correlated with altered gene expression in gunSOS1 compared to sigRep. Fumarate and aconitate will be described in more detail, as representative examples of the metabolites showing respectively increased or decreased content in gunSOS1 compared to sigRep.

KEGG enrichment of the RNA-seq data indicated increased expression of the gene encoding FUMARATE HYDRATASE CLASS II (FUM2, Enzyme Commission (EC) Number 4.2.1.2, Cre01.g020223) in gunSOS1 compared to sigRep. FUM is responsible for reversible stereospecific interconversion of malate to fumarate. However, specific isoforms of this protein act in defined pathways and may favour one direction over the other depending 
239 on the subcellular localisation and environment ${ }^{28}$. The mitochondrial isoform catalyses

240 hydration of fumarate to L-malate in the TCA cycle, while the cytosolic form catalyses 241 dehydration of L-malate to fumarate ${ }^{29,30}$. Two isoforms exist in $C$. reinhardtii, FUM1 242 encoded in locus Cre06.g254400 and FUM2. Based on the predicted subcellular localisation 243 (PredAlgo; http://lobosphaera.ibpc.fr/cgi-bin/predalgodb2.perl?page=main), FUM1 localizes 244 to the mitochondria, while FUM2 is assigned to the "other" compartment and is likely to be a 245 cytosolic protein. Pathway (KEGG) diagrams for the TCA cycle and pyruvate metabolism 246 can be found in Supplementary Fig. 6, showing integration of metabolite, RNA-seq and qRT247 PCR data. Comparison of the transcriptomes of gunSOS1 and sigRep also indicated increased 248 expression of the FUMARYLACETOACETASE gene (locus Cre17.g732802, EC 3.7.1.2) 249 involved in tyrosine catabolism. FUMARYLACETOACETASE catalyses hydrolysis of 4250 fumarylacetoacetate to acetoacetate and fumarate (Supplementary Fig. 7). Increased 251 expression of genes encoding FUM2 and FUMARYLACETOACETASE could explain accumulation of fumarate in gunSOS1 compared to sigRep.

Aconitate deficiency in gunSOS1 compared to sigRep can be explained by transcriptional downregulation of ACONITATE HYDRATASE (ACH1, Cre01.g042750, EC 4.2.1.3). ACH1 interconverts citrate and isocitrate, via cis-aconitate, in the TCA cycle (Supplementary Fig. 8) and is also involved in glyoxylate and dicarboxylate metabolism. At equilibrium, the reactants of the ACH1 reaction are present in the following ratio: 91\% citrate, $6 \%$ isocitrate and $3 \%$ aconitate. With the smallest pool of the three tricarboxylic acids, fluctuations in the level of aconitate are expected to be more pronounced compared to the other metabolites involved, especially citrate, whose content was similar in gunSOS1 and sigRep in the light (Supplementary Fig. 5). Taken together, our data indicate that altered fumarate and aconitate content in gunSOS1 compared to sigRep can be explained by altered expression of genes encoding enzymes involved in key metabolic processes affecting those metabolites.

Impaired ${ }^{1} \mathrm{O}_{2}$-signalling in gunSOS1 correlates with decreased expression of PSBP2, $M B S$, and $S A K 1$

The PSBP2 ${ }^{14}, \mathrm{MBS}^{16}$, and $\mathrm{SAK} 1^{15}$ proteins are required for ${ }^{1} \mathrm{O}_{2}$-induced chloroplast retrograde signalling in $C$. reinhardtii. Therefore, we determined the transcript levels of $P S B P 2, M B S$, and $S A K 1$, which were 8-, 9-, and nearly 41-fold lower, respectively, in gunSOS1 compared to sigRep (Fig. 7a). Decreased expression of SAK1 in gunSOS1 prompted 272 us to compare transcript levels of selected ${ }^{1} \mathrm{O}_{2}$-responsive genes between gunSOS1 and sak1, 
273 which lacks acclimation response to ${ }^{1} \mathrm{O}_{2}{ }^{15}$. SAK1 is a key regulator of the gene expression

274 response and its involvement in ${ }^{1} \mathrm{O}_{2}$-signalling was determined following treatment with rose

275 bengal ${ }^{15}$, which produces ${ }^{1} \mathrm{O}_{2}$ in the light. Nevertheless, based on our qRT-PCR analysis, all

276 tested genes had the same reduced inducibility in gunSOS1 compared to sigRep (Fig. 7b), as

277 it was observed in sakl compared to WT following ${ }^{1} \mathrm{O}_{2}$-exposure ${ }^{15}$. Among the genes that

278 were down-regulated in both gunSOS1 (Fig. 7b) and sak1 $1^{15}$ were two CYCLOPROPANE

279 FATTY ACID SYNTHASES, CFA1 and CFA2 (CPLD27), involved in lipid and sterol 280 metabolism, as well as the gene encoding SOUL1 heme-binding protein (Fig. 7b). It is 281 noteworthy that attenuated expression of both CFA1 and CFA2, as well as SOUL1, was also 282 observed in studies on a gpx 5 mutant $^{31}$. Furthermore, in agreement with the sakl phenotype 283 presented in Wakao, et al. ${ }^{15}$, gunSOS1 also showed decreases in transcript content of 284 PEPTIDE METHIONINE SULFOXIDE REDUCTASE (MSRA3), ALCOHOL 285 DEHYDROGENASE (ADH7), RETINALDEHYDE BINDING PROTEIN-RELATED 286 (RABPR2), and Cre01.g007300 encoding N287 ACETYLPHOSPHATIDYLETHANOLAMINE-HYDROLYZING PHOSPHOLIPASE D 288 (Fig. 7b). Thus, there is an overlap between the phenotypes of gunSOS1 and sak $1^{15}$ with 289 respect to their attenuated expression of genes induced during elevated ${ }^{1} \mathrm{O}_{2}$ (Fig. 7b).

291 Altered metabolite content affects ${ }^{1} \mathbf{O}_{2}$-signalling

292 We hypothesized that at least some of the metabolites showing increased or decreased 293 content in gunSOS1 compared to sigRep, attenuate or propagate ${ }^{1} \mathrm{O}_{2}$-signalling, respectively. 294 We selected fumarate and aconitate as representatives of either group. To test our hypothesis, 295 metabolites were added separately to the liquid cultures at empirically determined sublethal 296 concentrations, followed by incubation in the light to induce ${ }^{1} \mathrm{O}_{2}$ generation by Proto. 297 Subsequently, the $G P X 5_{c y t}$ and $G P X 5_{c p}$ transcript levels were used as readouts for the ${ }^{1} \mathrm{O}_{2}$ 298 signalling efficiency in gunSOS1 and sigRep.

299 Exogenous application of fumarate significantly $(P<0.001$, a detailed report from 300 two-way ANOVA analyses is presented in Supplementary Tables 4 and 5) attenuated 301 expression of both $G P X 5_{c y t}$ and $G P X 5_{c p}$ in a concentration-dependent manner in sigRep (Fig. 302 8a). As expected, fumarate did not affect GPX5 expression in gunSOS1. Application of 20 $303 \mu \mathrm{M}$ aconitate significantly $(P<0.0001)$ increased expression of GPX5 in gunSOS1 and even 304 further in sigRep compared to untreated cells $(P<0.0001)$, but $100 \mu \mathrm{M}$ had a quenching 305 effect on the GPX5 expression in sigRep (Fig. 8b, Supplementary Tables 6 and 7). This 306 indicates that aconitate up to a certain threshold concentration promotes ${ }^{1} \mathrm{O}_{2}$ signalling. 
Subsequently, we determined the expression of SAK1 upon feeding gunSOS1 and sigRep with aconitate. Exogenous application of aconitate at $20 \mu \mathrm{M}$ increased SAK1 transcript abundance in gunSOS1 more than 120 times compared to the untreated gunSOS1 control, and exceeded values observed in sigRep subjected to the same treatment by a factor of 5 (Fig. 8c). Due to the increased SAK1 expression in gunSOS1 upon feeding with aconitate, we also determined the expression of PSBP2 and $M B S$ in the same conditions. The $P S B P 2$ response to aconitate was similar to $S A K 1$ in terms of the increased expression (Fig. $8 \mathrm{c})$, but with less pronounced dependence on the metabolite concentration compared to SAK1. Although $20 \mu \mathrm{M}$ aconitate increased PSBP2 expression in gunSOS1 by a factor of 48 compared to the untreated control, it was still 2.7 times lower compared to sigRep subjected to the same treatment (Fig. 8c). Similarly to SAK1 and PSBP2, $20 \mu \mathrm{M}$ aconitate also increased expression of $M B S$ in gunSOS1, although values did not exceed those observed in sigRep subjected to the same treatment (Fig. 8c). Statistical analyses indicated that the effect of $20 \mu \mathrm{M}$ aconitate on SAK1, PSBP2 and MBS expression in sigRep and gunSOS1, interaction between mutants and aconitate, as well as the mutant-dependent expression of these genes were always significant $(P<0.001$, Supplementary Tables 8 -10).

\section{Specificity of the ${ }^{1} \mathrm{O}_{2}$-signalling pathway(s) attenuated in gunSOS1}

The profound effect of accumulating Tre6P on the transcriptome (Fig. 6a-c) and consequently the metabolism of gunSOS1 (Fig. 3 and Fig. 4) may have been indicative of a general photooxidative stress response being impaired in gunSOS1. To determine the specificity of this response, we compared gunSOS1 and sigRep in terms of the expression of selected genes that are known to be induced by various reactive species or conditions causing photooxidative stress other than ${ }^{1} \mathrm{O}_{2}$.

Upon $\mathrm{H}_{2} \mathrm{O}_{2}$ or organic tert-butyl hydroperoxide $\left(t\right.$-BOOH) treatment, Blaby, et al. ${ }^{32}$ observed an increase in the expression of the MSD3 gene, encoding plastid-localised $\mathrm{Mn}$ SUPEROXIDE DISMUTASE 3. In our studies, we did not observe an increase in MSD3 transcript in sigRep compared to WT (Fig. 9a), which shows that MSD3 expression is not inducible by ${ }^{1} \mathrm{O}_{2}$ produced by Proto. However, based on qRT-PCR analysis, a 9-fold increase was observed in gunSOS1 compared to sigRep (Fig. 9a, see also RNA-seq in Supplementary Data Set 2). Upon $\mathrm{H}_{2} \mathrm{O}_{2}$ or $t$-BOOH treatment, Urzica, et al. ${ }^{33}$ also observed induced expression of genes involved in the glutathione-ascorbate system, GDP-L-GALACTOSE PHOSPHORYLASE (VTC2) and DEHYDROSASCORBATE REDUCTASE (DHAR1). Based on our qRT-PCR results, VTC2 transcript content was not changed in sigRep compared to 
341 WT, but an increase was observed in gunSOS1 (Fig. 9b; Supplementary Data Set 2). DHAR1

342 expression was also stimulated in gunSOS1 compared to sigRep (Fig. 9c; Supplementary

343 Data Set 2).

344 Similarly to sak1 following treatment with rose bengal ${ }^{15}$, we observed increased 345 expression of GLUTATHIONE S-TRANSFERASE (GSTS1) in gunSOS1 compared to sigRep 346 (Fig. 9d, Supplementary Data Set 2). However, in another study increased expression of 347 GSTS1 was shown after treatment with acrolein, which suggests its transcriptional induction 348 by reactive electrophile species (RES) ${ }^{34}$. Acrolein was also shown to induce FSD1 encoding 349 Fe SUPEROXIDE DISMUTASE (FeSOD ${ }^{34}$, which was expressed both in sigRep and 350 gunSOS1 (Supplementary Data Set 1), and no significant difference could be observed in 351 DEG analysis (Supplementary Data Set 2) or qRT-PCR (Fig. 9e). It can be concluded that, 352 despite impaired ${ }^{1} \mathrm{O}_{2}$-signalling, gunSOS1 retained the ability to respond to photooxidative stress caused by other ROS such as $\mathrm{H}_{2} \mathrm{O}_{2}$, organic peroxides, or RES.

\section{Discussion}

356

357

ROS are formed as a by-product of biological redox reactions ${ }^{35}$ mostly in the mitochondria or chloroplasts $^{36,37}$. Although excess ROS production can cause oxidative damage to cell components, ROS or the oxidation products play an important role in the signal transduction processes.

Different retrograde signalling pathways have been proposed to involve also TBSintermediates in plants and green algae (reviewed $\mathrm{in}^{38,39}$ ). While involvement of $\mathrm{Mg}$ porphyrins in chloroplast retrograde signalling is now excluded ${ }^{40,41}$, in the present study we have demonstrated that ${ }^{1} \mathrm{O}_{2}$ produced by the photosensitizing activity of Proto in the light, but not Proto itself, triggers signalling cascades that alter nuclear gene expression. Using strains that endogenously accumulate Proto, we identified several mutants that are impaired in ${ }^{1} \mathrm{O}_{2}$ signalling. Endogenous accumulation of Proto in mutants is advantageous for studying ${ }^{1} \mathrm{O}_{2}$ signalling, because it eliminates the need for exogenous application of TBS or carotenoid biosynthesis inhibitors, as well as photosensitizers, such as rose bengal or neutral red (Fischer et al., 2004) to induce ${ }^{1} \mathrm{O}_{2}$ generation. The lack of chlorophyll provides another advantage, because such mutants do not have functional photosynthetic electron transport, so that ${ }^{1} \mathrm{O}_{2}$ production in photosynthesis is avoided. The accumulating Proto is thus the dominant source 372 of generated ${ }^{1} \mathrm{O}_{2}$.

The ${ }^{1} \mathrm{O}_{2}$-induced signalling phenotype in the gunSOS1 mutant was due to a lesion in 374 the T6PP1 gene. We demonstrated that T6PP1 is a functional phosphatase capable of 
375 dephosphorylating Tre6P. T6PP1 is the only representative of the classical plant TPP gene

376 family in C. reinhardtii, contrasting with the large $T P P$ gene families in angiosperms ${ }^{42}$. In

377 addition, C. reinhardtii has one representative of the class I TREHALOSE-6-PHOSPHATE

378 SYNTHASE (TPS) family (Cre16.g662350, hereafter T6PS1), and two members of the class II

379 TPS family (Cre06.g278221, here T6PSP1 and Cre16.g686200, here T6PSP2). Both class I

380 and class II TPS proteins have glucosyltransferase and TPP-like domains, but only the class I

381 TPS proteins have demonstrated TPS activity ${ }^{43-45}$. T6PP1 belongs to the haloacid

382 dehalogenase superfamily of proteins and contains the characteristic DXDX(T/V) active site

383 motif $-{ }_{107} \mathrm{DYDGT}_{112}$ - in which the initial Asp residue forms a phospho-acyl intermediate

384 during catalysis ${ }^{46}$. T6PSP1 also contains the complete active site motif ( ${ }_{592} \mathrm{DYDGT}_{597}$ ), so we

385 cannot exclude the possibility that the T6PSP1 protein in C. reinhardtii has TPP activity,

386 which could explain the increased content of trehalose in gunSOS1 compared to sigRep.

387 Although the intracellular localizations of these proteins have not been determined

388 experimentally in C. reinhardtii, PredAlgo analysis indicated possible localization of T6PP1

389 and T6PSP1 in mitochondria, while T6PS1 and T6PSP2 are likely to be cytosolic proteins,

390 because they were not assigned to any specific organelle.

391 Correlation between T6PP1 (Fig. 2b) and GPX5 (Fig. 1b, c) expression indicates that

392 low Tre6P content is required for efficient ${ }^{1} \mathrm{O}_{2}$-signalling. However, the apparent negative

393 effect of Tre6P on ${ }^{1} \mathrm{O}_{2}$-signalling is not direct and involves a complex metabolic

394 reprogramming (Fig. 10). Study on the contrasting phenotypes between $A$. thaliana

395 overexpressing bacterial TPS, TPP, or TREHALOSE PHOSPHATE HYDROLASE (TPH)

396 pointed to Tre6P, rather than trehalose, playing a signalling function ${ }^{25}$. Tre6P was shown to

397 be highly correlated with sucrose, leading to the proposal that it functions as a signal of

398 sucrose status $^{23}$. Tre6P was also shown to inhibit starch degradation in . thaliana $^{47,48}$, which

399 is also true in Tre6P-acumulating gunSOS1 mutant of C. reinhardtii.

400 It was demonstrated that Tre6P acts as an inhibitor of SUCROSE-NON-

401 FERMENTING (SNF)-RELATED PROTEIN KINASE1 (SnRK1) in developing tissues and

402 that this is dependent on a so-far unidentified protein factor ${ }^{49-51}$. Tre6P also inhibits the

403 activation of SnRK1 by SnRK1-activating kinases/geminivirus Rep interacting kinases ${ }^{52}$.

404 SnRK1 belongs to the AMPK-SNF1-SnRK family of protein kinases, which is represented in

405 all eukaryotes ${ }^{53}$. In plants, SnRK1 plays a central role in energy and metabolic homeostasis,

406 and is activated during energy deficient conditions caused by stresses like nutrient starvation,

407 pathogen attack, or $\operatorname{ROS}^{54}$. Baena-González, et al. ${ }^{55}$ established approximately 1000 genes as

408 markers of SnRK1 in A. thaliana, which indicates an extensive SnRK1-dependent 
409 transcriptional reprogramming. In C. reinhardtii, involvement of various SnRKs in responses 410 to stress was observed during sulphur ${ }^{56,57}$ and nitrogen deprivation ${ }^{58}$, or cold stress ${ }^{59}$.

411 Genome-wide analysis revealed the existence of 21 genes as potential orthologues of the 412 plant $\operatorname{SnRK} \alpha, \beta$ and $\gamma / \beta \gamma$ subunits in $C$. reinhardtii. It was suggested that the proteins 413 encoded by these genes play the same role in cell survival and stress response in $C$. 414 reinhardtii as SnRKs in land plants ${ }^{58}$.

415 In analogy to A. thaliana, altered SnRKs activity was shown to cause metabolic 416 remodelling also in algae ${ }^{59}$. In our study, the majority of changes to gene expression observed 417 in gunSOS1, relative to sigRep, originates from accumulation of Tre6P and, based on 418 previous studies, this leads to inhibition of one or more SnRKs (Fig. 10). Our analysis 419 showed that altered gene expression in gunSOS1 relative to sigRep (Supplementary Fig. 6-8) 420 is directly responsible for altered fumarate and aconitate content in gunSOS1 compared to 421 sigRep. Thus, Tre6P accumulating in gunSOS1 is not directly involved in ${ }^{1} \mathrm{O}_{2}$-signalling but 422 controls other processes in the cell which affect ${ }^{1} \mathrm{O}_{2}$-signalling more directly (Fig. 10).

423 We demonstrated that accumulation of fumarate is responsible for attenuating ${ }^{1} \mathrm{O}_{2}$ 424 signalling (Fig. 8a, Fig. 10). Fumarate is a well-recognized oncometabolite in mammalian 425 cells $^{60}$, associated with development of tumours by competitive inhibition of 2-oxoglutarate426 dependent oxygenases, including HYPOXIA INDUCIBLE FACTOR (HIF) hydroxylases, 427 leading to stabilisation of HIF and activation of oncogenic HIF-dependent pathways ${ }^{61}$. In 428 fact, accumulation of fumarate in human cells was linked to an aggressive variant of 429 hereditary kidney cancer ${ }^{62}$. In mice, fumarate was also shown to directly modify some 430 proteins by succination of cysteine residues to form 2 -succinocysteine derivatives ${ }^{63}$. 431 Succination of three cysteines crucial for iron-sulphur cluster binding was identified in 432 mitochondrial ACONITASE2 (ACO2) in a fumarate hydratase 1 knockout (Fh1KO) mouse 433 embryonic fibroblast (MEF) cell line ${ }^{63}$. Analysis of tryptic peptides derived from ACO2 in 434 Fh1KO MEFs, indicated succination of Cys385. Another tryptic peptide of ACO2 was 435 identified as a mixture of two isomers in Fh1KO MEFs, which showed succination at Cys451 436 or Cys $448^{63}$. Succination of these cysteine residues in ACO2 leads to its inhibition ${ }^{63}$. 437 Equivalent cysteine residues are conserved in C. reinhardtii ACH1, which explains the 438 aconitate deficiency in gunSOS1 (Fig. 4a).

439 The gunSOS1 mutant showed decreased levels of aconitate compared to sigRep, while 440 exogenous application of this metabolite to gunSOS1 restored ${ }^{1} \mathrm{O}_{2}$-signalling. Mammalian $441 \mathrm{ACO} 2$ is thought to be a mitochondrial oxidative stress sensor, which requires an active $442\left[\mathrm{Fe}_{4} \mathrm{~S}_{4}\right]^{2+}$ cluster $^{64}$. It is, therefore, proposed that accumulation of fumarate deactivates ACH1 
443 and reduces the aconitate content in gunSOS1, thereby impairing oxidative stress sensing and $444{ }^{1} \mathrm{O}_{2}$-signalling. Diminished expression of $A C H 1$ in gunSOS1 relative to sigRep 445 (Supplementary Fig. 8a, b) may be an additional factor adding to the aconitate depletion in 446 gunSOS1.

447 Results obtained in our study indicate that there is a metabolic signature conditioning $448{ }^{1} \mathrm{O}_{2}$-signalling. A mechanism in which the chloroplast controls nuclear gene expression via 449 metabolite levels was postulated before ${ }^{12,65}$. It was shown that altered chloroplast redox 450 conditions result in changes in the metabolome in A. thaliana, reflected also by the 451 reallocation of energy resources ${ }^{66}$. Importantly, although our study shows that the metabolic 452 configuration of the cell is essential for ${ }^{1} \mathrm{O}_{2}$-signalling, this does not exclude the involvement 453 of protein components in this signalling network (Fig. 10). On the contrary, our findings 454 support the direct involvement of proteins in ${ }^{1} \mathrm{O}_{2}$-signalling, but we demonstrated that expression of $P S B P 2^{14}, M B S^{16}$, and $S A K 1^{15}$ depends on the metabolic status (Fig. 7a and Fig. 456 8a, b). The overlap between the gunSOS1 and the SAK1-dependent transcriptomes (Fig. 7b) 457 as well as other defined ROS or RES-dependent signalling pathways being functional (Fig. 458 9a-e) indicate specificity of the impaired ${ }^{1} \mathrm{O}_{2}$-signalling in gunSOS1. Our results indicate that 459 fumarate and aconitate affect the expression of $P S B P, M B S$, or $S A K 1$, which subsequently 460 convey information about the metabolic status of the cell to the nucleus and trigger specific responses (Fig. 10). It is not clear yet how the specificity of the signalling is achieved, because the mechanisms by which metabolites affect the expression of these proteins, as well as downstream components of these signalling pathways, remain unknown. Additionally, the protein components involved in ${ }^{1} \mathrm{O}_{2}$-signalling may be subject to additional regulatory mechanisms, such as phosphorylation, as proposed for SAK1 by Wakao, et al. ${ }^{15}$.

We have shown that retrograde signalling triggered by ${ }^{1} \mathrm{O}_{2}$, which is mostly generated in the chloroplast, strictly depends on the mitochondrial metabolic status. PROLYL-tRNA SYNTHETASE (PRORS1) in A. thaliana, a component of the organellar gene expression machinery indicated that mitochondria may contribute to chloroplast retrograde signalling ${ }^{67}$. PRORS1 is targeted both to the plastid and to mitochondria, but downregulation of specific photosynthesis-associated nuclear genes was observed only in a plastidial prpl11 and mitochondrial mrpl11 double mutant, but not in prpll1 or mrpl11 single mutants ${ }^{67}$. Another study showed that the ROS-dependent signals from chloroplast and mitochondria are integrated at the RADICAL-INDUCED CELL DEATH 1 (RCD1) protein located in the nucleus $^{68}$. RCD1 suppresses transcription factors ANAC013 and ANAC017, which mediate the ROS-signal from mitochondria, while chloroplast regulation of RCD1 takes place through 
477 3'-phosphoadenosine 5'-phosphate. It was proposed that RCD1 may function at the 478 intersection of mitochondrial and chloroplastic retrograde signalling pathways ${ }^{68}$.

479 The retrograde signalling pathways identified so far assume the existence of separate 480 signalling routes. However, chloroplast retrograde signalling involves a network of different 481 but inter-connected mechanisms, and observation of specific signalling pathways within the 482 network depends on the experimental conditions. Pharmacological or genetic interventions 483 that disrupt specific pathways can be used to characterize alternative pathways. In the 484 manuscript presented here, we report that the metabolic status of the cell, reflecting levels of 485 Tre6P, and components of the mitochondrial TCA cycle are integral factors in the ${ }^{1} \mathrm{O}_{2}$ 486 dependent retrograde signalling network in C. reinhardtii.

\section{Methods}

\section{Chlamydomonas reinhardtii cultures and genetic manipulations}

490 All strains were cultivated heterotrophically in Tris-acetate-phosphate (TAP) medium in the dark. Additionally, starch measurements were performed in TAP, Tris-phosphate (TP) medium without acetate, or nitrogen-depleted (TAP-N) medium. Experiments were performed in TAP medium in the dark or upon a shift to $20 \mu \mathrm{mol}$ photons $\mathrm{m}^{-2} \mathrm{~s}^{-1}$ light. Three strains used in this study were described elsewhere, chlD-1 in von Gromoff, et al. ${ }^{17}$, chlD$1 / G U N 4$ in Brzezowski, et al. ${ }^{18}$, and wild type $(4 \mathrm{~A}+)$ in Dent, et al. ${ }^{69}$.

To generate the GPX5-ARS2 construct (Supplementary Fig. 2), the GPX5 regulatory region $(G P X 55$, RR) was amplified by PCR using forward and reverse primers carrying XhoI and EcoRV restrictions sites, respectively (Supplementary Table 11). The obtained fragment was subcloned and ligated between the XhoI/EcoRV sites of the pSL18 vector ${ }^{70}$, replacing the existing promoter region of the gene encoding the PSAD and positioning GPX5 5 'RR in reverse orientation with respect to the paromomycin resistance cassette (paro ${ }^{\mathrm{R}, 71}$. The DNA fragment carrying paro ${ }^{\mathrm{R}}$ and $G P X 55^{\prime}$, RR was excised by restriction digestion with $503 \mathrm{KpnI}$ and EcoRV and ligated into the corresponding restriction sites of pJD54 ${ }^{72}$, which carries a copy of the promoterless version of the gene encoding ARYLSULFATASE 2 (ARS2), which allowed ARS2 expression to be controlled by the GPX5 5'RR (Supplementary Fig. 2). The GPX5-ARS2 construct was verified by sequencing.

507 The sigRep strain was produced in the chlD-1/GUN4 background by transformation with a GPX5-ARS2 construct. Transformant selection was performed on TAP agar plates with $10 \mu \mathrm{g} \mathrm{mL}^{-1}$ paromomycin in dark, followed by a screen for GPX5-ARS2 inducibility by ${ }^{1} \mathrm{O}_{2}$. 510 The sigRep strain showed low ARS2 activity in the dark and high activity in the light (Fig. 
511 1a) and was selected for further applications. The gunSOS1 mutant was generated by random 512 insertional mutagenesis performed on sigRep using a bleomycin resistance cassette (ble ${ }^{\mathrm{R}}$ ) 513 isolated from the pMS188 vector $^{73}$ using NotI and KpnI. Following mutagenesis, selection 514 was performed on TAP agar plates containing $15 \mu \mathrm{g} \mathrm{mL}^{-1}$ zeocin in the dark. More than 800 515 obtained colonies were screened for decreased or not detectable ARS2 activity in the light 516 and nine mutants that showed impaired inducibility of GPX5-ARS expression in light were 517 selected for further analysis. Restriction enzyme site-directed amplification $\mathrm{PCR}^{74}$ allowed 518 us to identify the genomic DNA flanking the ble ${ }^{R}$ insertion sites in five out of the nine 519 mutants. Only analysis of the gunSOS1 mutant is presented here. Rescue of gunSOS1 (t6pp1) 520 was conducted with 5242 bp fragment of genomic DNA carrying 3241 bp T6PP1 amplified 521 by PCR (see Supplementary Table 11 for primers) using bacterial artificial chromosome 522 PTQ5987 (Clemson University Genomics Institute, Clemson, SC, USA) as a template. The 523 amplified DNA fragment included $1500 \mathrm{bp}$ upstream and $501 \mathrm{bp}$ downstream of the 524 annotated T6PP1. Wild-type T6PP1 was introduced into gunSOS1 by co-transformation with 525 a spectinomycin resistance cassette isolated from the pALM32 vector ${ }^{75}$ with AleI and KpnI 526 endonucleases. Transformant selection was performed on TAP agar plates supplemented with $527100 \mu \mathrm{g} \mathrm{mL}^{-1}$ spectinomycin in the dark. All genetic transformations were performed by 528 electroporation.

\section{Arylsulfatase activity assay}

531 To assess the level of GPX5-ARS2 expression in transformed cells, enzymatic assays 532 for arylsulfatase activity ${ }^{76}$ were performed essentially as described before ${ }^{14}$. Cells were 533 spotted onto agar-solidified TAP medium plates and cultured in either light or dark 534 conditions. Arylsulfatase is expected to be secreted into the medium if GPX5-ARS2 is 535 expressed. After removal of the cells, plates were flooded with detection solution containing $5360.1 \mathrm{mg} \mathrm{mL}^{-1}$ 2-naphthyl sulfate (potassium salt; Santa Cruz Biotechnology, Inc., Dallas, TX, 537 USA) as a chromogenic substrate coupled with $1 \mathrm{mg} \mathrm{mL}^{-1}$ tetrazotized-o-dianisidine chloride 538 (Fast Blue B salt, Santa Cruz Biotechnology, Inc., Dallas, TX, USA). Following $1 \mathrm{~h}$ 539 incubation, purple spots appearing on the agar plates identified expressed ARS2.

\section{Analysis of protoporphyrin IX content}

542 The Proto content was analysed by High Pressure Liquid Chromatography (HPLC) 543 essentially as described in Czarnecki, et al. ${ }^{77}$, with modified sample preparation for $C$. 544 reinhardtii ${ }^{18}$. In short, cultures were grown in the dark and transferred to $20 \mu \mathrm{mol}$ photons $\mathrm{m}^{-}$ 
$545{ }^{2} \mathrm{~s}^{-1}$ light for $2 \mathrm{~h}$. Samples containing $1.2 \times 10^{8}$ cells were centrifuged at $3000 \mathrm{x} g$ for $5 \mathrm{~min}$ at $5464^{\circ} \mathrm{C}$ and the pellets were snap-frozen in liquid $\mathrm{N}_{2}$. Proto was extracted in cold $\left(-20^{\circ} \mathrm{C}\right)$ 547 acetone/0.1 M NH $4 \mathrm{OH}(9 / 1, \mathrm{v} / \mathrm{v})$ in a three-step cycle of resuspension and centrifugation. 548 Proto analysis was performed using a Nova-Pak C18 column (Waters, $3.9 \times 150 \mathrm{~mm}, 4 \mu \mathrm{m}$, 549 at $20^{\circ} \mathrm{C}$ ). The results were normalized to $\mathrm{pmol} / 10^{6}$ cells.

550

\section{RNA isolation, qRT-PCR, and RNA-seq}

552 The total RNA was isolated, after a shift from dark to $20 \mu \mathrm{mol}$ photons $\mathrm{m}^{-2} \mathrm{~s}^{-1}$ light for $2 \mathrm{~h}$, 553 using TRIzol Reagent (Thermo Fisher Scientific, Waltham, MA, USA), according to the 554 manufacturer's protocol. RNA quality was assessed by electrophoresis on a $1 \%(\mathrm{w} / \mathrm{v})$ agarose 555 gel, while quantity was determined using a Nanodrop 2000 (Thermo Fischer Scientific, 556 Waltham, MA). Aliquots of $2 \mu \mathrm{g}$ RNA were treated with DNase and RiboLock RNase 557 inhibitor (Thermo Fisher Scientific, Waltham, MA, USA) and used to synthesize cDNA with 558 RevertAid Reverse Transcriptase (Thermo Fisher Scientific, Waltham, MA, USA) and 559 oligo(dT) $)_{18}$ primer. Transcript analysis by qRT-PCR were performed using $2 \times$ ChamQ 560 Universal SYBR qPCR Master Mix (Viazyme Biotech Co., Ltd., Nanjing, China) and a 561 CFX96-C1000 96-well plate thermocycler (Bio-Rad, Hercules, CA, USA), the 18S rRNA 562 was used as a reference gene ${ }^{22}$. All primers were designed using Primer3Plus 563 (http://primer3plus.com), and are listed in Supplementary Table 11.

RNA-sequencing was performed on total RNA samples isolated from biological triplicates of sigRep and gunSOS1 after the shift from dark to $20 \mu \mathrm{mol}$ photons $\mathrm{m}^{-2} \mathrm{~s}^{-1}$ light for $2 \mathrm{~h}$. Library preparation, sequencing, and analysis services were commercially provided by Novogene Europe (Novogene (UK) Company Ltd., Cambridge, UK) and performed using an Illumina NovaSeq 6000 platform operated in 150 bp pair end sequencing mode, with a sequencing depth of 20 million reads per sample. Six gigabases of sequencing data per library was filtered for high-quality reads, which were mapped to the $C$. reinhardtii v5.5

571 (Department of Energy JGI). Estimated expression was obtained in fragments per kilobase of transcript sequence per millions base pairs sequenced (FPKM). Biological replicates were averaged to obtain sample expression estimate. Differential expression analysis between two mutants (three biological replicates per mutant) was performed using DESeq2.v1.20.0, which provides statistical routines for determining differential expression using a model based on the negative binomial distribution. The resulting $p$ values were adjusted using the Benjamini

577 and Hochberg's approach for controlling the False Discovery Rate (FDR). Genes with a $578 \log _{2}$ FoldChange $\geq 2$ and adjusted $p$ value (padj) $<0.05$ found by DESeq2.v1.20.0 were 
579 defined as differentially expressed (DEGs). Gene Ontology (GO) analysis and Kyoto 580 Encyclopedia of Genes and Genomes (KEGG; www.genome.jp/kegg/pathway.html) pathway 581 analysis were conducted to identify DEGs at the biologically functional level using 582 clusterProfiler $\mathrm{R}$ package. GO terms and KEGG pathways with a padj $<0.05$ were 583 considered to be significantly enriched.

\section{Metabolite analysis}

586 Cultures were grown in TAP in the dark until they reached mid-log phase of $3 \times 10^{6}$ cells mL$^{-}$

$587{ }^{1}$. Samples were always normalized to contain $6 \times 10^{7}$ cells, all centrifugation steps were 588 carried at $3000 \times g$ at $4^{\circ} \mathrm{C}$ for $5 \mathrm{~min}$, followed by snap-freezing in liquid $\mathrm{N}_{2}$. Samples from 589 dark conditions were collected immediately, while the remainder of each culture was 590 transferred to $20 \mu \mathrm{mol}$ photons $\mathrm{m}^{-2} \mathrm{~s}^{-1}$ light. Subsequent sampling took place at $15-\mathrm{min}$ 591 intervals, up to $1 \mathrm{~h}$. Metabolites were extracted using chloroform-methanol as described by 592 Lunn et al. (2006). Tre6P, other phosphorylated intermediates and organic acids were 593 measured by anion-exchange HPLC coupled to tandem mass spectrometry (LC-MS/MS) as 594 described in Lunn et al. (2006) with modifications as described in $^{78}$. Sugars and sugar 595 alcohols were measured by LC-MS/MS as described in ${ }^{79}$. Obtained results were normalized 596 to $\mathrm{pmol} / 10^{6}$ cells.

597 For starch analysis, cultures were grown in TAP in the dark until they reached mid$598 \log$ phase of $3 \times 10^{6}$ cells $\mathrm{mL}^{-1}$. For starch determination cultures were either transferred in 599 TAP to the light $\left(20 \mu \mathrm{mol}\right.$ photons $\left.\mathrm{m}^{-2} \mathrm{~s}^{-1}\right)$ for $2 \mathrm{~h}$ before harvesting, or centrifuged cells were 600 resuspended in Tris-phosphate (TP) medium (without acetate), keeping the same cell 601 concentration, and transferred back to the dark for $24 \mathrm{~h}$. Subsequently, cultures were 602 transferred to $20 \mu \mathrm{mol}$ photons $\mathrm{m}^{-2} \mathrm{~s}^{-1}$ for $2 \mathrm{~h}$ before harvesting. A similar protocol was 603 applied for starch analysis in TAP devoid of N (TAP-N), except that cells were cultivated in 604 TAP-N for 3 days in the dark, followed by exposure to $20 \mu \mathrm{mol}$ photons $\mathrm{m}^{-2} \mathrm{~s}^{-1}$ for $2 \mathrm{~h}$. For 605 each sampling, $5 \times 10^{6}$ cells were centrifuged and lyophilized. Starch content was determined 606 by enzymatic degradation and glucose quantification following the protocol of DuBois, et al. $607 \quad 80$.

608

\section{Protein extraction and immunoblot analysis}

610 Cultures were grown in the dark, followed by a transfer to $20 \mu \mathrm{mol}$ photons $\mathrm{m}^{-2} \mathrm{~s}^{-1}$ for $3 \mathrm{~h}$. 611 Cells were pelleted by centrifugation and total proteins were extracted in $400 \mu \mathrm{L}$ buffer 612 containing: $56 \mathrm{mM} \mathrm{Na}_{2} \mathrm{CO}_{3}, 56 \mathrm{mM} \mathrm{DTT}, 2 \%(\mathrm{w} / \mathrm{v}) \mathrm{SDS}, 12 \%(\mathrm{w} / \mathrm{v})$ sucrose and $2 \mathrm{mM}$ 
613 EDTA, pH 8.0. Proteins were quantified using Pierce ${ }^{\mathrm{TM}}$ BCA Protein Assay Kit (Thermo 614 Fisher Scientific, Waltham, MA, USA) and separated by SDS-PAGE on a 12\% 615 polyacrylamide gel, followed by transfer by electroblotting to nitrocellulose membrane (GE 616 Healthcare, Chicago, IL, USA). T6PP1 was detected using a rabbit crude antiserum (dilution 617 1:500) raised by inoculation with a T6PP1-specific peptide (VEWSKSDSNGWRAKPC) 618 against $C$. reinhardtii T6PP1 (calculated MW $42 \mathrm{kDa}$ ). GPX5 was detected with a 619 commercially available antibody (AS15 2882, dilution 1:1000) obtained from Agrisera 620 (Vännäs, Sweden). CHLI1 antibody (PHY5510S, dilution 1:1000) was purchased from 621 PhytoAB (San Jose, CA, USA). For application, all antibodies were diluted in CrossDown 622 buffer (AppliChem, AppliChem GmbH, Darmstadt, Germany). The secondary antibody 623 (AS09 602, goat anti-rabbit IgG, dilution 1:10,000) conjugated to horseradish peroxidase was 624 obtained from Agrisera. The immunoblotting signals were detected using a CCD camera 625 (Intas Biopharmaceuticals, Ahmedabad, India) after application of enhanced 626 chemiluminescence detection kit (Clarity ${ }^{\mathrm{TM}}$ Western ECL Substrate; Bio-Rad, Hercules, CA, 627 USA.

\section{Chemical treatments of $C$. reinhardtii cells}

630 Fumarate (sodium fumarate dibasic) and aconitate (cis-aconitic acid) were obtained from 631 Sigma-Aldrich (Sigma-Aldrich Chemie $\mathrm{GmbH}$, Taufkirchen, Germany), dissolved in $\mathrm{H}_{2} \mathrm{O}$ to 632 a stock solution of $100 \mathrm{mM}$ and added individually to the mid-log phase $\left(3 \times 10^{6}\right.$ cells $\left.\mathrm{mL}^{-1}\right)$ 633 cultures to final concentrations of 20,50 , and $100 \mu \mathrm{M}$, followed by exposure to light for $2 \mathrm{~h}$.

\section{Data availability statement}

636 The data that support the findings of this study are available from the corresponding author 637 upon reasonable request.

\section{References}

6401 Johanningmeier, U. \& Howell, S. H. Regulation of light-harvesting chlorophyll-binding 641 protein mRNA accumulation in Chlamydomonas reinhardii. Possible involvement of chlorophyll synthesis precursors. J. Biol. Chem. 259, 3541-3549 (1984).

2 Oelmüller, R. Photooxidative destruction of chloroplasts and its effect on nuclear gene expression and extraplastidic enzyme levels. Photochem. Photobiol. 49, 229-239, doi:10.1111/j.1751-1097.1989.tb04101.x (1989).

3 Susek, R. E., Ausubel, F. M. \& Chory, J. Signal transduction mutants of Arabidopsis uncouple nuclear $\mathrm{CAB}$ and RBCS gene expression from chloroplast development. Cell 74, 787-799 (1993). 


\footnotetext{
6494 Oster, U., Brunner, H. \& Rudiger, W. The greening process in cress seedlings .5. Possible interference of chlorophyll precursors, accumulated after thujaplicin treatment, with lightregulated expression of Lhc genes. J. Photoch. Photobio. B 36, 255-261, doi:10.1016/s10111344(96)07388-5 (1996).

5 Lydon, J. \& Duke, S. O. Porphyrin synthesis is required for photobleaching activity of the para-nitrosubstituted diphenyl ether herbicides. Pestic. Biochem. Physiol. 31, 74-83, doi:10.1016/0048-3575(88)90031-4 (1988).

6 Triantaphylides, C. et al. Singlet oxygen is the major reactive oxygen species involved in photooxidative damage to plants. Plant Physiol. 148, 960-968, doi:10.1104/pp.108.125690 (2008).

7 op den Camp, R. G. L. et al. Rapid induction of distinct stress responses after the release of singlet oxygen in Arabidopsis. Plant Cell 15, 2320-2332, doi:10.1105/tpc.014662 (2003).

8 Baruah, A., Simkova, K., Apel, K. \& Laloi, C. Arabidopsis mutants reveal multiple singlet oxygen signaling pathways involved in stress response and development. Plant Mol. Biol. 70, 547-563, doi:10.1007/s11103-009-9491-0 (2009).

9 Gorman, A. A. \& Rodgers, M. A. J. Current perspectives of singlet oxygen detection in biological environments. J. Photoch. Photobio. B 14, 159-176 (1992).

10 Beck, C. F. Signaling pathways from the chloroplast to the nucleus. Planta 222, 743-756, doi:10.1007/s00425-005-0021-2 (2005).

11 Sies, H. \& Menck, C. F. M. Singlet oxygen induced DNA damage. Mutat. Res. 275, 367-375 (1992).

12 Pfannschmidt, T. Plastidial retrograde signalling - a true "plastid factor" or just metabolite signatures? Trends Plant Sci. 15, 427-435, doi:10.1016/j.tplants.2010.05.009 (2010).

13 Kim, C. et al. Chloroplasts of Arabidopsis are the source and a primary target of a plantspecific programmed cell death signaling pathway. Plant Cell 24, 3026-3039, doi:10.1105/tpc.112.100479 (2012).

14 Brzezowski, P., Wilson, K. E. \& Gray, G. R. The PSBP2 protein of Chlamydomonas reinhardtii is required for singlet oxygen-dependent signaling. Planta 236, 1289-1303, doi:10.1007/s00425-012-1683-1 (2012).

15 Wakao, S. et al. Phosphoprotein SAK1 is a regulator of acclimation to singlet oxygen in Chlamydomonas reinhardtii. eLife 3, e02286, doi:10.7554/eLife.02286 (2014).

16 Shao, N., Duan, G. Y. \& Bock, R. A mediator of singlet oxygen responses in Chlamydomonas reinhardtii and Arabidopsis identified by a luciferase-based genetic screen in algal cells. Plant Cell 25, 4209-4226, doi:10.1105/tpc.113.117390 (2013).

17 von Gromoff, E. D., Alawady, A., Meinecke, L., Grimm, B. \& Beck, C. F. Heme, a plastidderived regulator of nuclear gene expression in Chlamydomonas. Plant Cell 20, 552-567, doi:10.1105/tpc.107.054650 (2008).

18 Brzezowski, P. et al. The GUN4 protein plays a regulatory role in tetrapyrrole biosynthesis and chloroplast-to-nucleus signalling in Chlamydomonas reinhardtii. Plant J. 79, 285-298, doi:10.1111/tpj.12560 (2014).

19 Fischer, B. B., Krieger-Liszkay, A. \& Eggen, R. I. L. Photosensitizers neutral red (Type I) and rose bengal (Type II) cause light-dependent toxicity in Chlamydomonas reinhardtii and induce the Gpxh gene via increased singlet oxygen formation. Environ. Sci. Technol. 38, 6307-6313, doi:10.1021/es049673y (2004).

20 Leisinger, U. et al. The glutathione peroxidase homologous gene from Chlamydomonas reinhardtii is transcriptionally up-regulated by singlet oxygen. Plant Mol. Biol. 46, 395-408 (2001).

21 Fischer, B. B., Il Eggen, R. \& Niyogi, K. K. Characterization of singlet oxygen-accumulating mutants isolated in a screen for altered oxidative stress response in Chlamydomonas reinhardtii. BMC Plant Biol. 10, doi:279 10.1186/1471-2229-10-279 (2010).

22 Fischer, B. B. et al. Function and regulation of the glutathione peroxidase homologous gene GPXH/GPX5 in Chlamydomonas reinhardtii. Plant Mol. Biol. 71, 569-583, doi:10.1007/s11103-009-9540-8 (2009).
} 


\begin{tabular}{|c|c|c|}
\hline 757 & 42 & $\begin{array}{l}\text { Vandesteene, L. et al. Expansive evolution of the trehalose-6-phosphate phosphatase gene } \\
\text { family in Arabidonsis. Plant Physiol. 160.884-896 doi:10.1104/pn.112.201400 (2012). }\end{array}$ \\
\hline 758 & & amily in Arabidopsis. Plant Physiol. 160, 884-896, do1:10.1104/pp. $112.201400(2012)$. \\
\hline $\begin{array}{l}759 \\
760\end{array}$ & 43 & $\begin{array}{l}\text { Ramon, M. et al. Extensive expression regulation and lack of heterologous enzymatic activity } \\
\text { of the Class II trehalose metabolism proteins from Arabidopsis thaliana. Plant Cell Environ. }\end{array}$ \\
\hline 761 & & 2, 1015-1032, doi:10.1111/j.1365-3040.2009.01985.x (2009). \\
\hline 762 & 44 & Vandesteene, L., Ramon, M., Le Roy, K., Van Dijck, P. \& Rolland, F. A single active \\
\hline 763 & & ehalose-6-P synthase (TPS) and a family of putative regulatory TPS-like proteins in \\
\hline 764 & & rabidopsis. Mol. Plant 3, 406-419, doi:10.1093/mp/ssp114 (2010). \\
\hline 765 & 45 & Delorge, I., Figueroa, C. M., Feil, R., Lunn, J. E. \& Van Dijck, P. Trehalose-6-phosphate \\
\hline 766 & & hase 1 is not the only active TPS in Arabidopsis thaliana. Biochem. J. 466, 283-290, \\
\hline 767 & & $\mathrm{i}: 10.1042 / \mathrm{bj} 20141322$ (2015). \\
\hline 768 & 46 & Collet, J. F., Stroobant, V., Pirard, M., Delpierre, G. \& Van \\
\hline 769 & & hosphotransferases phosphorylated on an aspartate residue in an amino-terminal \\
\hline 770 & & DXDX(T/V) motif. J. Biol. Chem. 273, 14107-14112, doi:10.1074/jbc.273.23.14107 (1998). \\
\hline 771 & 47 & Martins, M. C. et al. Feedback inhibition of starch degradation in Arabidopsis leaves \\
\hline 772 & & mediated by trehalose 6-phosphate. Plant Physiol. 163, 1142-1163, \\
\hline 773 & & doi:10.1104/pp.113.226787 (2013). \\
\hline 774 & 48 & Dos Anjos, L. et al. Feedback regulation by trehalose 6-phosphate slows down starch \\
\hline 775 & & Jilzation Defow the rate mat wou \\
\hline 776 & & Plant direct 2, e00078, doi:10.1002/pld3.78 (2018). \\
\hline 777 & 49 & Zhang, Y. et al. Inhibition of SNF1-related protein kinase1 activity and regulation \\
\hline 778 & & by trehalose-6-phosphate. Plant Physiol. 149, \\
\hline 779 & & i:10.1104/pp.108.133934 (2009). \\
\hline 780 & 50 & Debast, S. et al. Altering trehalose-6-phosphate content in transgenic potato tubers affects \\
\hline 781 & & erc \\
\hline 782 & & 771, doi:10.1104/pp.111.179s \\
\hline 783 & 51 & Nunes, C. et al. Inhibition of SnRK1 by metabolites: tissue-dependent effects and cooperative \\
\hline 784 & & \\
\hline 785 & & Biochem. 63, 89-98, doi:10 \\
\hline 786 & 52 & Zhai, Z. et al. Trehalose 6-Phosphate Positively Regulates Fatty Acid Synthesis by Stabilizing \\
\hline 787 & & RINKLED1 Plant Cell $\mathbf{3 0}, 2$ \\
\hline 788 & 53 & Baena-González, E. \& Lunn, J. E. SnRK1 and trehalose 6-phosphate - two ancient pathways \\
\hline 789 & & converge to regulate plant metabolism and growth. Curr. Opin. Plant Biol. 55, 52-5 \\
\hline 790 & & ii $202001010(2020)$ \\
\hline 791 & 54 & Depaepe, T. et al. At the Crossroads of Survival and Death: The Reactive Oxygen Species- \\
\hline 792 & & Ethylene-Sugar Triad and the Unfolded Protein Response. Trends Plant Sci. 26, 338-351, \\
\hline 793 & & i:10.1016/j.tplants.2020.12.007 (2021). \\
\hline 794 & 55 & Baena-González, E., Rolland, F., Thevelein, J. M. \& Sheen, J. A central inte \\
\hline 795 & & tworks in plant stress and energy signalling. Nature 448, \\
\hline 796 & & i:10.1038/nature06069 (2007). \\
\hline 797 & 56 & Gonzalez-Ballester, D., Pollock, S. V., Pootakham, W. \& Grossman, A. R. The central role o \\
\hline 798 & & a SNRK2 kinase in sulfur deprivation responses. Plant Physiol. 147, 216-227, \\
\hline 799 & & doi:10.1104/pp.108.116137 (2008). \\
\hline 800 & 57 & González-Ballester, D. et al. RNA-seq analysis of sulfur-deprived Chlamydomonas cells \\
\hline 801 & & vival. Plant Cell \\
\hline 802 & & $\mathrm{i}: 10.1105 / \mathrm{tpc} .109 .071167$ (2010) \\
\hline 803 & 58 & Colina, F. et al. Genome-wide identification and characterization of CKIN/SnRK gene family \\
\hline 804 & & $018-35625-8(2010$ (2) \\
\hline 805 & 59 & Valledor, L., Furuhashi, T., Hanak, A. M. \& Weckwerth, W. Systemic cold stress adaptation \\
\hline 806 & & of Chlamydomonas reinhardtii. Mol. Cell. Proteomics 12, \\
\hline 807 & & doi:10.1074/mcp.M112.026765 (2013). \\
\hline 808 & 60 & ng, M., Soga, T., Pollard, P. J. \& \\
\hline 80 & & \\
\hline
\end{tabular}


81061 O'Flaherty, L. et al. Dysregulation of hypoxia pathways in fumarate hydratase-deficient cells is independent of defective mitochondrial metabolism. Hum. Mol. Genet. 19, 3844-3851, doi:10.1093/hmg/ddq305 (2010).

62 Shanmugasundaram, K. et al. The oncometabolite fumarate promotes pseudohypoxia through noncanonical activation of NF- $\mathrm{kB}$ signaling. J. Biol. Chem. 289, 24691-24699, doi:10.1074/jbc.M114.568162 (2014).

63 Ternette, N. et al. Inhibition of mitochondrial aconitase by succination in fumarate hydratase deficiency. Cell reports 3, 689-700, doi:10.1016/j.celrep.2013.02.013 (2013).

64 Lloyd, S. J., Lauble, H., Prasad, G. S. \& Stout, C. D. The mechanism of aconitase: 1.8 A resolution crystal structure of the S642a:citrate complex. Protein Sci. 8, 2655-2662, doi:10.1110/ps.8.12.2655 (1999).

65 Kleine, T., Voigt, C. \& Leister, D. Plastid signalling to the nucleus: messengers still lost in the mists? Trends Genet. 25, 185-190, doi:10.1016/j.tig.2009.02.004 (2009).

66 Bräutigam, K. et al. Dynamic plastid redox signals integrate gene expression and metabolism to induce distinct metabolic states in photosynthetic acclimation in Arabidopsis. Plant Cell 21, 2715-2732, doi:10.1105/tpc.108.062018 (2009).

67 Pesaresi, P. et al. Nuclear photosynthetic gene expression is synergistically modulated by rates of protein synthesis in chloroplasts and mitochondria. Plant Cell 18, 970-991, doi:10.1105/tpc.105.039073 (2006).

68 Shapiguzov, A. et al. Arabidopsis RCD1 coordinates chloroplast and mitochondrial functions through interaction with ANAC transcription factors. eLife 8, doi:10.7554/eLife.43284 (2019).

69 Dent, R. M., Haglund, C. M., Chin, B. L., Kobayashi, M. C. \& Niyogi, K. K. Functional genomics of eukaryotic photosynthesis using insertional mutagenesis of Chlamydomonas reinhardtii. Plant Physiol. 137, 545-556, doi:10.1104/pp.104.055244 (2005).

70 Depege, N., Bellafiore, S. \& Rochaix, J. D. Role of chloroplast protein kinase Stt7 in LHCII phosphorylation and state transition in Chlamydomonas. Science 299, 1572-1575 (2003).

71 Sizova, I., Fuhrmann, M. \& Hegemann, P. A Streptomyces rimosus aphVIII gene coding for a new type phosphotransferase provides stable antibiotic resistance to Chlamydomonas reinhardtii. Gene 277, 221-229 (2001).

72 Davies, J. P., Weeks, D. P. \& Grossman, A. R. Expression of the arylsulfatase gene from the B2-tubulin promoter in Chlamydomonas reinhardtii. Nucleic Acids Res. 20, 2959-2965 (1992).

73 Schroda, M., Beck, C. F. \& Vallon, O. Sequence elements within an HSP70 promoter counteract transcriptional transgene silencing in Chlamydomonas. Plant J. 31, 445-455, doi:10.1046/j.1365-313X.2002.01371.x (2002).

74 Gonzalez-Ballester, D., de Montaigu, A., Galvan, A. \& Fernandez, E. Restriction enzyme site-directed amplification PCR: A tool to identify regions flanking a marker DNA. Anal. Biochem. 340, 330-335, doi:10.1016/j.ab.2005.01.031 (2005).

75 Meslet-Cladière, L. \& Vallon, O. Novel shuttle markers for nuclear transformation of the green alga Chlamydomonas reinhardtii. Eukaryot. Cell 10, 1670-1678, doi:10.1128/ec.0504311 (2011).

76 Ohresser, M., Matagne, R. F. \& Loppes, R. Expression of the arylsulphatase reporter gene under the control of the nitl promoter in Chlamydomonas reinhardtii. Curr. Genet. 31, 264271 (1997).

77 Czarnecki, O., Peter, E. \& Grimm, B. in Chloroplast research in Arabidopsis: Methods and Protocols, Vol II Vol. 775 357-385 (2011).

78 Figueroa, C. M. et al. Trehalose 6-phosphate coordinates organic and amino acid metabolism with carbon availability. Plant J. 85, 410-423, doi:10.1111/tpj.13114 (2016).

79 Fichtner, F. et al. Trehalose 6-phosphate is involved in triggering axillary bud outgrowth in garden pea (Pisum sativum L.). Plant J. 92, 611-623, doi:10.1111/tpj.13705 (2017).

80 DuBois, M., Gilles, K. A., Hamilton, J. K., Rebers, P. A. \& Smith, F. Colorimetric Method for Determination of Sugars and Related Substances. Analytical chemistry 28, 350-356, doi:10.1021/ac60111a017 (1956). 
$86481 \quad$ Johnson, X. \& Alric, J. Central carbon metabolism and electron transport in Chlamydomonas

\section{Acknowledgements}

871 This work was supported by the Deutsche Forschungsgemeinschaft (DFG-GR 936/20-2 to

\section{Author contributions}

876 P.B. designed the research. P.B. and W.A.Y. performed most of the experiments. R.F. and 877 J.E.L. performed the LC-MS/MS measurements. M.S.-S. and X.J. performed the starch 878 quantification. P.B., B.G., and J.E.L. analysed the data. P.B. wrote the manuscript. P.B., 879 B.G., J.E.L., and X.J. revised the manuscript. All authors discussed the results and commented upon the manuscript.

\section{Competing interests}

883 The authors declare no competing financial interests.

\section{Additional information}

886 Supplementary information accompanies this manuscript:

Supplementary Figure 1. Schematic representation of tetrapyrrole biosynthesis pathway.

Supplementary Figure 2. Schematic representation of the GPX5-ARS2 reporter gene construct.

890 Supplementary Figure 3. Analysis of Proto content in gunSOS1.

891 Supplementary Figure 4. Rescue of the ${ }^{1} \mathrm{O}_{2}$-signalling in gunSOS1 by introduction of the 892 wild-type T6PP1.

893 Supplementary Figure 5. Metabolites content in gunSOS1 compared to sigRep.

894 Supplementary Figure 6. Accumulation of fumarate in gunSOS1 compared to sigRep could 895 be explained by increased expression of FUMARATE HYDRATASE 2.

896 Supplementary Figure 7. Accumulation of fumarate in gunSOS1 compared to sigRep could 897 be explained by increased expression of FUMARYLACETOACETASE. 
898 Supplementary Figure 8. Deficiency in aconitate in gunSOS1 compared to sigRep could be 899 explained by decreased expression of ACONITATE HYDRATASE 1.

900 Supplementary Table 1. Metabolites analyzed in gunSOS1 compared to sigRep (LC$901 \mathrm{MS} / \mathrm{MS})$.

902 Supplementary Table 2. Impact of accumulating metabolites on cell metabolism.

903 Supplementary Table 3. Impact of deficient metabolites on cell metabolism.

904 Supplementary Table 4. Statistical analyses for $G P X 5_{c y t}$ expression following treatment 905 with fumarate, presented in Figure 7a.

906 Supplementary Table 5. Statistical analyses for $G P X 5_{c p}$ expression following treatment with 907 fumarate, presented in Figure 7a.

908 Supplementary Table 6. Statistical analyses for $G P X 5_{c y t}$ expression following treatment 909 with aconitate, presented in Figure $7 \mathrm{~b}$.

910 Supplementary Table 7. Statistical analyses for $G P X 5_{c p}$ expression following treatment with 911 aconitate, presented in Figure $7 \mathrm{~b}$.

912 Supplementary Table 8. Statistical analyses for $S A K 1$ expression following treatment with 913 aconitate, presented in Figure 7c.

914 Supplementary Table 9. Statistical analyses for PSBP2 expression following treatment with 915 aconitate, presented in Figure 7c.

916 Supplementary Table 10. Statistical analyses for $M B S$ expression following treatment with 917 aconitate, presented in Figure 7c.

918 Supplementary Table 11. Primers used in this study.

919 Supplementary Data Set 1. Co-expression analysis

920 Supplementary Data Set 2. DEG analysis

921 Supplementary Data Set 3. KEGG analysis 
a

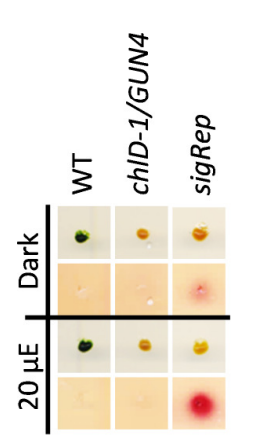

b

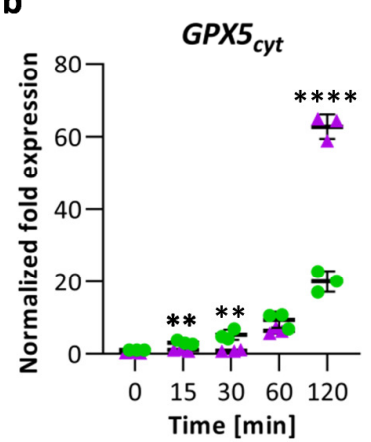

e

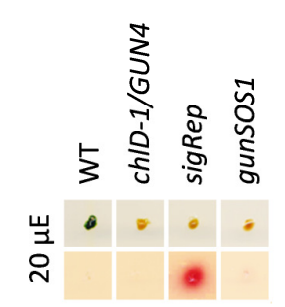

g

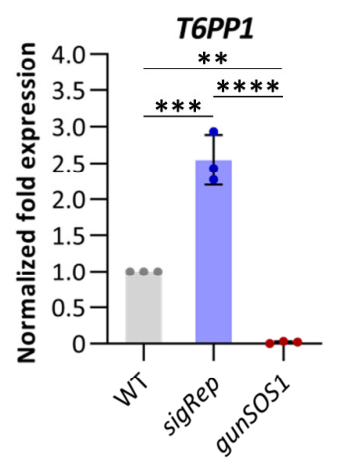

h

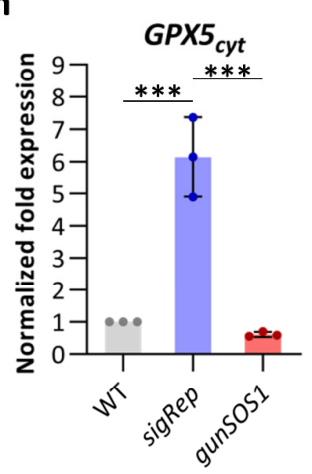

chID-1/GUN4

sigRep

gunsos1

WT

chID-1
PAR [ $\mu \mathrm{mol}$ photons $\mathrm{m}^{-2} \mathrm{~s}^{-1}$ ]

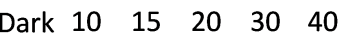

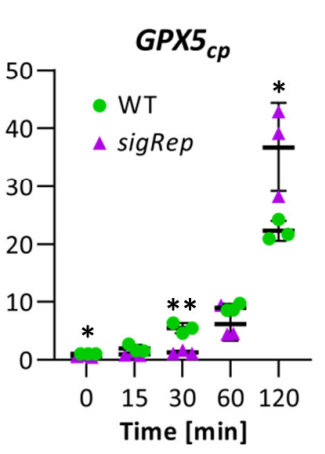

c

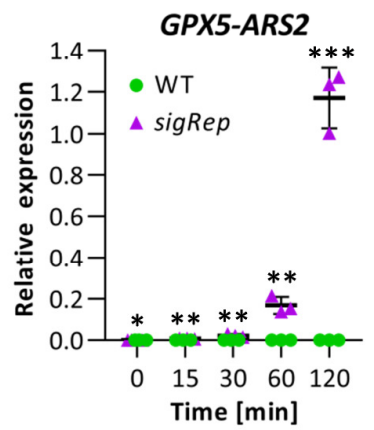

f
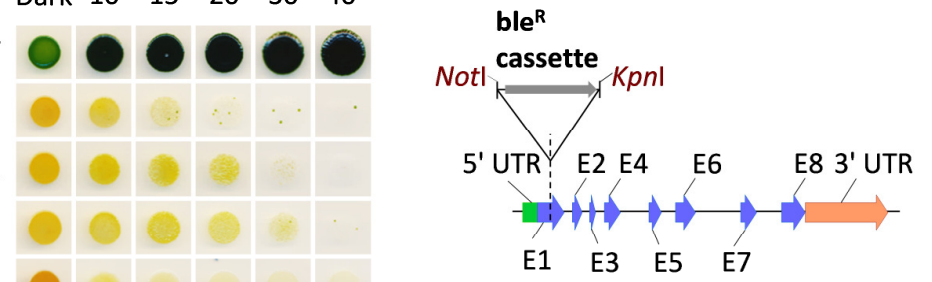

924

925

926

927

928

929

930

931

932

933

934

935 
936 Figure 1. Generation and initial characterization of the mutant impaired in ${ }^{1} \mathbf{O}_{2}$ 937 signalling. a Arylsulfatase (ARS2) assay for selection of the strain expressing GPX5-ARS2 938 reporter gene in a ${ }^{1} \mathrm{O}_{2}$-inducible manner. The mutant selected for further applications was 939 named signalling Reporter (sigRep). WT and chlD-1/GUN4 do not carry GPX5-ARS2 and 940 were used as negative controls in ARS2 assay. b Expression kinetics of cytosolic $\left(G P X 5_{c y t}\right)$ 941 and chloroplast GPX5 $\left(G P X 5_{c p}\right)$ in sigRep compared to WT upon exposure to light; the 942 values were normalized to WT in dark. c Expression of GPX5-ARS2 in sigRep positively 943 correlates with time of exposure to light. WT was used as a negative control. d ARS2 activity 944 assay following mutagenesis of sigRep. Screen was performed to identify mutants not 945 expressing GPX5-ARS2 compared to sigRep in the same conditions. The mutant selected for 946 further applications was named genomes uncoupled Singlet $\underline{\text { Oxygen }}$ Signalling $\underline{1}$ (gunSOS1).

947 WT and chlD-1/GUN4 were used as negative controls. e Strains with impaired ${ }^{1} \mathrm{O}_{2}$-signalling 948 demonstrated increased sensitivity to light compared to sigRep and chlD-1/GUN4. Higher 949 light tolerance of chlD-1/GUN4 and sigRep compared to chlD-1 is also visible. f T6PP1 950 (Cre12.g497750) gene model. The insertion (ble ${ }^{\mathrm{R}}$ ) was identified in the first exon of $3241 \mathrm{bp}$ 951 T6PP1. $\mathbf{g}$ Expression of T6PP1 in gunSOS1 compared to sigRep. $\mathbf{h}$ Expression of $G P X 5_{c y t}$ 952 and GPX5 $5_{c p}$ in gunSOS1 compared to sigRep. i Expression of GPX5-ARS2 in gunSOS1 953 compared to sigRep. WT was used as a negative control. Transcript analyses were performed 954 by qRT-PCR on biological triplicates, values were calculated either as a normalized fold 955 expression $\left(2^{-\Delta \Delta \mathrm{Ct}} ; \mathrm{WT}=1\right)$ or relative expression $\left(2^{\Delta \mathrm{Ct}}\right)$. For $\mathrm{b}$ and $\mathrm{c}$, horizontal bars represent 956 the calculated mean $(n=3)$, vertical error bars represent calculated $\pm \mathrm{SD}$; significant 957 differences were calculated using two-tailed Student's $t$-test and are indicated by asterisks 958 (non-significant not shown), ${ }^{*} P<0.05, * * P<0.01, * * * P<0.001$, and $* * * * P<0.0001$. For $959 \mathrm{~g}$-i, error bars indicate calculated $\pm \mathrm{SD}$; one-way ANOVA, pair-wise comparison with the 960 Tukey's post-hoc test (non-significant not shown), ${ }^{*} P<0.05, * * P<0.01, * * * P<0.001$, and $961 * * * * P<0.0001$. 
a

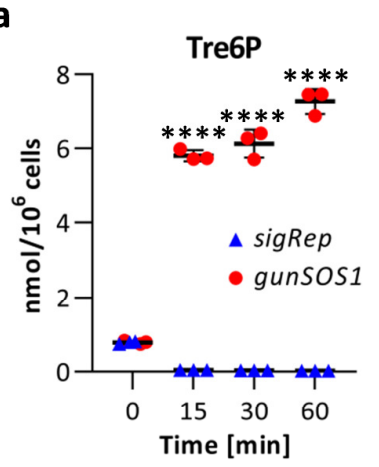

C

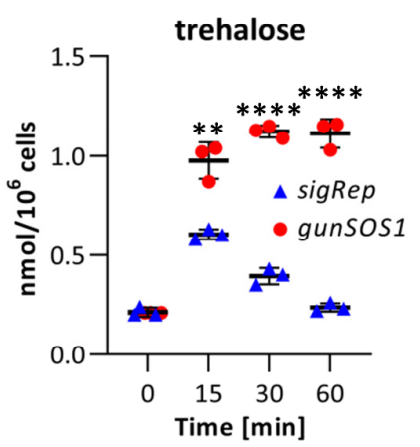

b

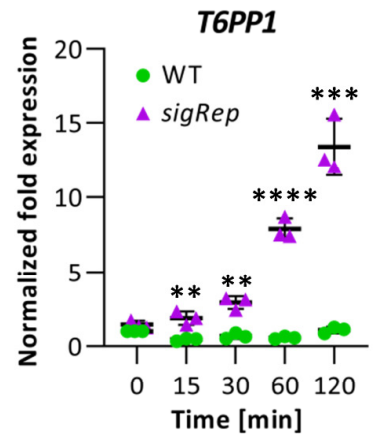

d

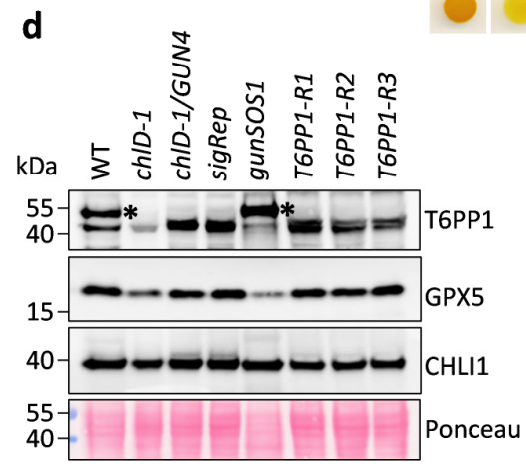

e

PAR [ $\mu$ mol photons $\mathrm{m}^{-2} \mathrm{~s}^{-1}$ ] Dark $10 \quad 15 \quad 20 \quad 30 \quad 40$

0000001

ChID-1

chID-1/GUN4

sigRep

gunSOS1

T6PP1-R1

T6PP1-R2

T6PP1-R3

\section{Figure 2. $C$. reinhardtii $\mathrm{T6PP1}$ is a functional phosphatase induced during} photooxidative stress. a Kinetics of Tre6P accumulation in gunSOS1 compared to sigRep upon transfer from dark to light. b Kinetics of T6PP1 expression upon transfer from dark to light in gunSOS1 compared to WT indicate inducibility by photooxidative stress rather than light; values were calculated as a normalized fold expression $\left(2^{-\Delta \Delta C t}\right.$; WT $\left.=1\right)$. c Higher content of trehalose in gunSOS1 compared to sigRep upon transfer from dark to light may be resulting from the function of T6PS/T6PP1. d Immuno-blot analysis of T6PP1 (calculated MW of $42 \mathrm{kDa}$ ) and GPX5 content in gunSOS1 compared to sigRep and strains rescued with wild-type copy of T6PP1. The CHLI1 content was used as a loading control. The unspecific immunoreaction is indicated by an asterisk. $\mathbf{e}$ Light sensitivity examination showed that the strains with rescued ${ }^{1} \mathrm{O}_{2}$-signalling have an increased tolerance to light compared to gunSOS1. Experiments in a, b and c were performed in biological triplicates $(n=3)$ horizontal bars represent the calculated mean, vertical error bars represent $\pm \mathrm{SD}$; significant differences were calculated using two-tailed Student's $t$-test and are indicated by asterisks (non-significant not shown), ${ }^{*} P<0.05,{ }^{* *} P<0.01,{ }^{* * *} P<0.001$, and $* * * * P<0.0001$. 
a
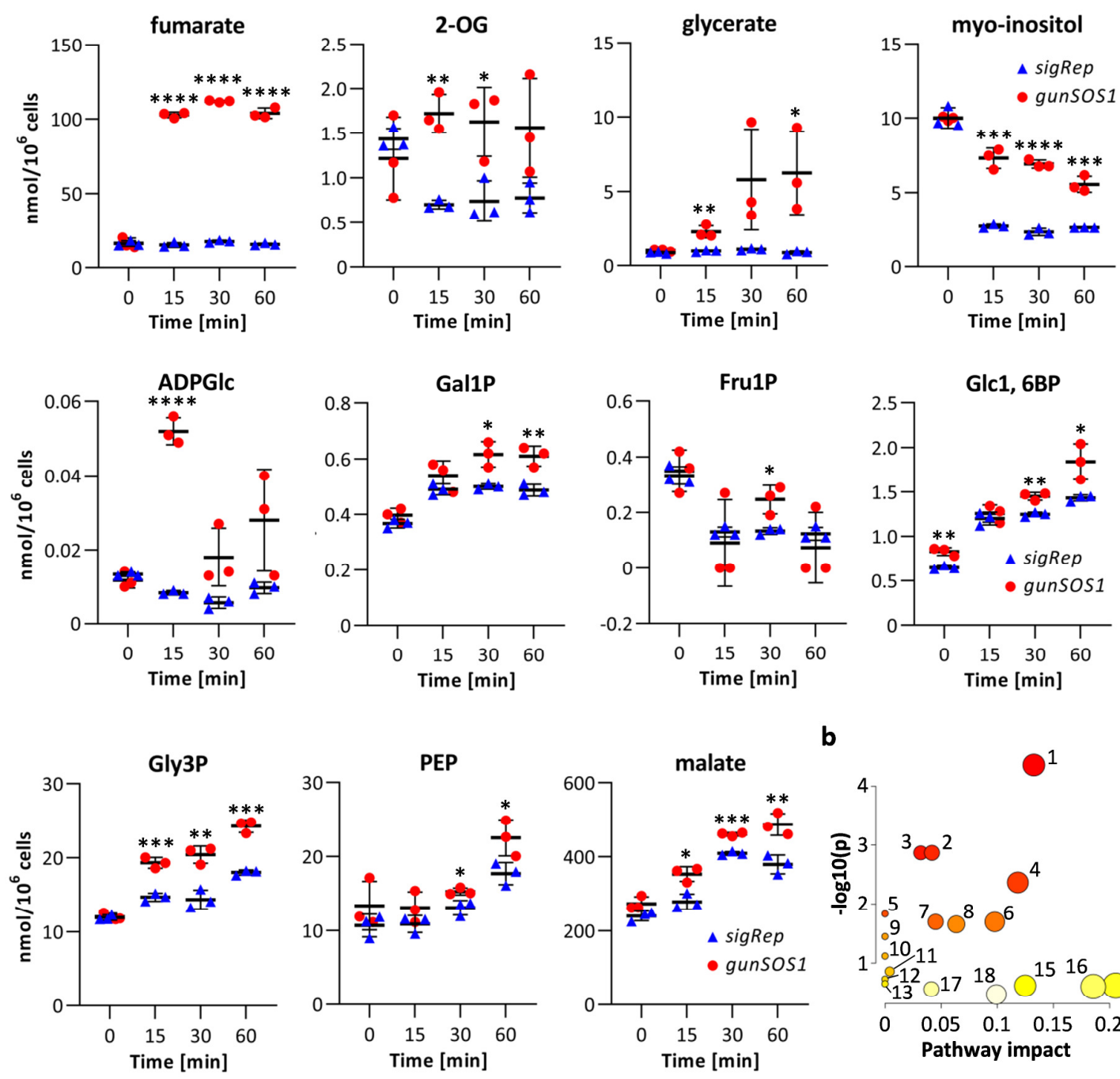

b 
992 Figure 3. Metabolites with significantly increased content in gunSOS1 compared to 993 sigRep in the light. a LC-MS/MS analysis; fumaric acid (fumarate), oxoglutaric acid (2-OG), 994 myo-Inositol, ADP-glucose (ADPGlc), galactose 1-phosphate (Gal1P), fructose 1-phosphate 995 (Fru1P), alpha-D-Glucose 1,6-bisphosphate (Glc1, 6BP), glycerol 3-phosphate (Gly3P), 996 phosphoenolpyruvic acid (PEP), L-Malic acid (malate). Measurements were performed in 997 biological triplicates and are presented as mean $(n=3)$, horizontal bars represent the 998 calculated mean, vertical error bars represent calculated $\pm \mathrm{SD}$; significant differences were 999 calculated using two-tailed Student's $t$-test and are indicated by asterisks (non-significant not 1000 shown), $* P<0.05, * * P<0.01, * * * P<0.001$, and $* * * * P<0.0001$. b Impact of 1001 accumulating metabolites on cell metabolism analysed using MetaboAnalyst 5.0 1002 (https://www.metaboanalyst.ca), sorted by the $P$ value; 1. Tricarboxylic acid cycle (TCA 1003 cycle; $P=4.41 \mathrm{E}-05)$, 2. Pyruvate metabolism $(P=0.00136)$, 3. Starch and sucrose 1004 metabolism $(P=0.00136), 4$. Glyoxylate and dicarboxylate metabolism $(P=0.00429), 5$. 1005 Arginine biosynthesis $(P=0.01473), 6$. Alanine, aspartate and glutamate metabolism $(P=$ 1006 0.02019), 7. Glycerolipid metabolism $(P=0.02019)$, 8. Carbon fixation in photosynthetic 1007 organisms $(P=0.02217), 9$. Galactose metabolism $(P=0.03566), 10$. Amino sugar and 1008 nucleotide sugar metabolism $(P=0.07616), 11$. Fructose and mannose metabolism $(P=$ 1009 0.13874), 12. Tyrosine metabolism $(P=0.1872), 13$. Phenylalanine, tyrosine and tryptophan 1010 biosynthesis $(P=0.22414), 14$. Inositol phosphate metabolism $(P=0.24202), 15$. Glycolysis 1011 / Gluconeogenesis $(P=0.24202), 16$. Phosphatidylinositol signaling system $(P=0.25082)$, 1012 17. Glycine, serine and threonine metabolism $(P=0.27665)$, 18. Glycerophospholipid 1013 metabolism $(P=0.34157)$; detailed results are presented in Supplementary Table 2. 

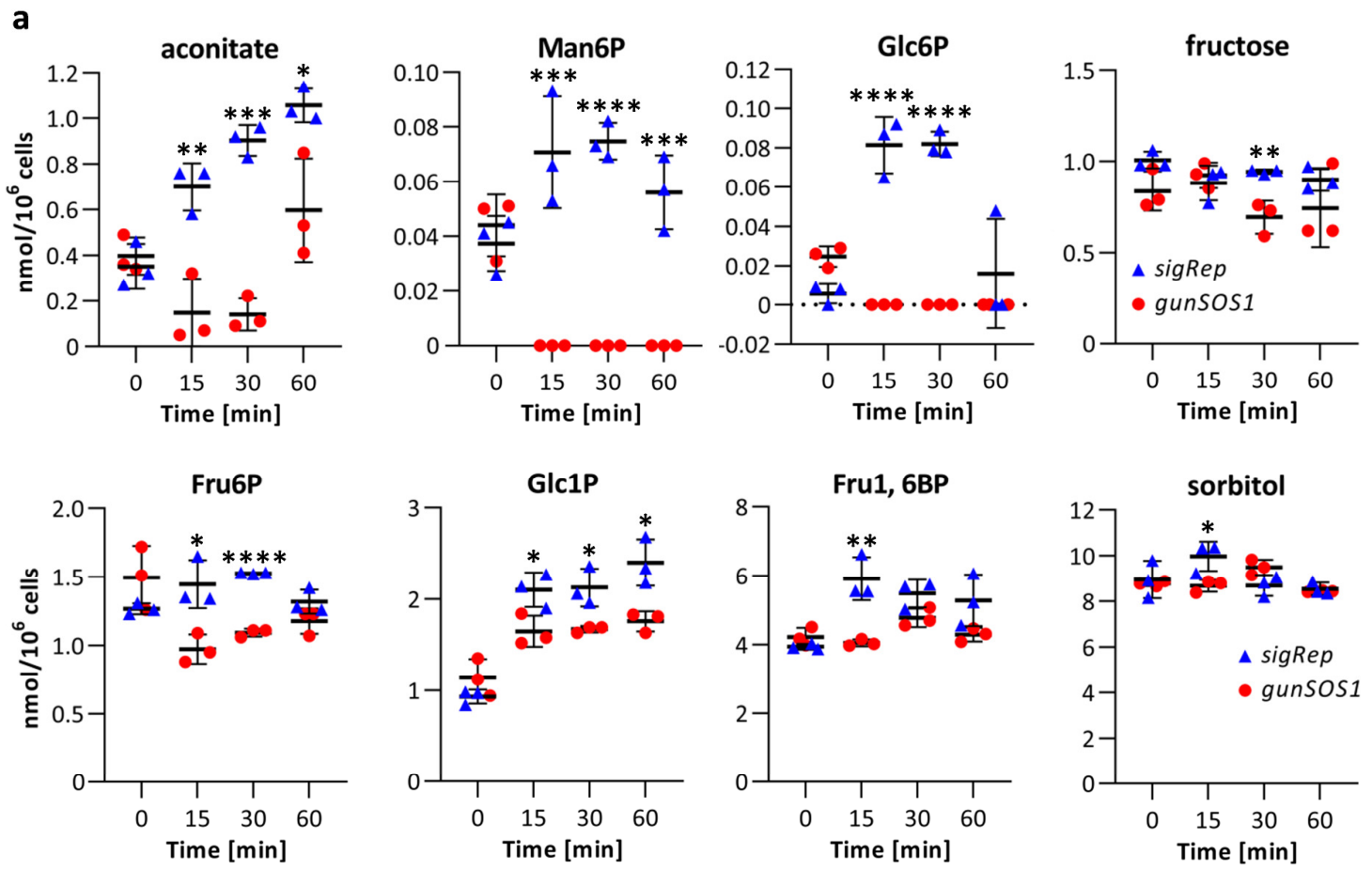

b

1015

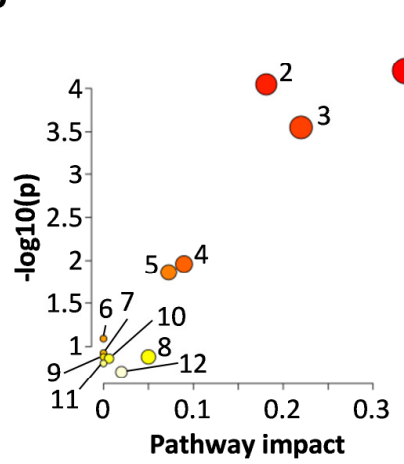

c

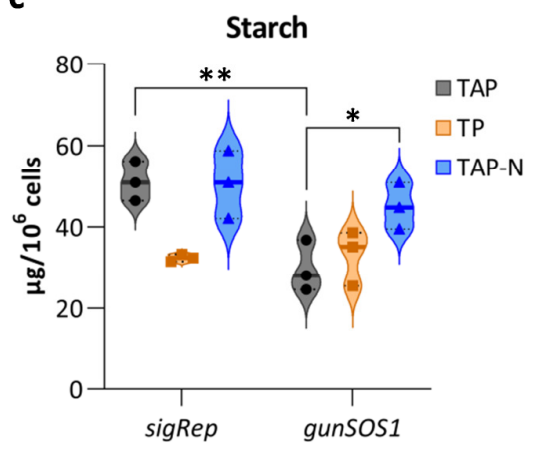

1016

1017

1018

1019

1020

1021

1022

1023

1024

1025

1026 
1027 Figure 4. Metabolites with significantly decreased content in gunSOS1 compared to 1028 sigRep in the light. a LC-MS/MS analysis; cis-Aconitic acid (aconitate), mannose 61029 phosphate (Man6P), Glucose 6-phosphate (Glc6P), D-fructose (frictose), Fructose 61030 phosphate (Fru6P), Glucose 1-phosphate (Glc1P), Fructose 1,6-bisphosphate (Fru1, 6BP), 1031 sorbitol. Measurements were performed in biological triplicates and are presented as mean $(n$ $1032=3$ ), horizontal bars represent the calculated mean, vertical error bars represent calculated $1033 \pm \mathrm{SD}$; significant differences were calculated using two-tailed Student's $t$-test and are 1034 indicated by asterisks (non-significant not shown), ${ }^{*} P<0.05$, ${ }^{*} P<0.01$, ${ }^{* * *} P<0.001$, and $1035 * * * * P<0.0001$. b Impact of deficient metabolites on cell metabolism analysed using 1036 MetaboAnalyst 5.0 (https://www.metaboanalyst.ca), sorted by the $P$ value; 1 . Fructose and 1037 mannose metabolism $(P=6.23 \mathrm{E}-05), 2$. Amino sugar and nucleotide sugar metabolism $(P=$ 1038 8.99E-05), 3. Starch and sucrose metabolism $(P=0.000282)$, 4. Glycolysis / 1039 Gluconeogenesis $(P=0.01094), 5$. Galactose metabolism $(P=0.013767), 6$. Pentose and 1040 glucuronate interconversions $(P=0.081139), 7$. Pentose phosphate pathway $(P=0.1195), 8$. 1041 Tricarboxylic acid cycle (TCA cycle; $P=0.13197)$, 9. Glycerolipid metabolism $(P=$ 1042 0.13197), 10. Carbon fixation in photosynthetic organisms $(P=0.13815)$, 11 . Inositol 1043 phosphate metabolism $(P=0.15646), 12$. Glyoxylate and dicarboxylate metabolism $(P=$ 1044 0.19785); detailed results are presented in Supplementary Table 3. c Starch accumulation in 1045 gunSOS1 compared to sigRep. Measurements were performed in biological triplicates $(n=3)$; 1046 median is shown as a center line; upper and lower quartiles are shown as dotted lines. 1047 Significant differences were calculated using two-tailed Student's $t$-test and are indicated by 1048 asterisks (non-significant not shown), ${ }^{*} P<0.05, * * P<0.01$. 


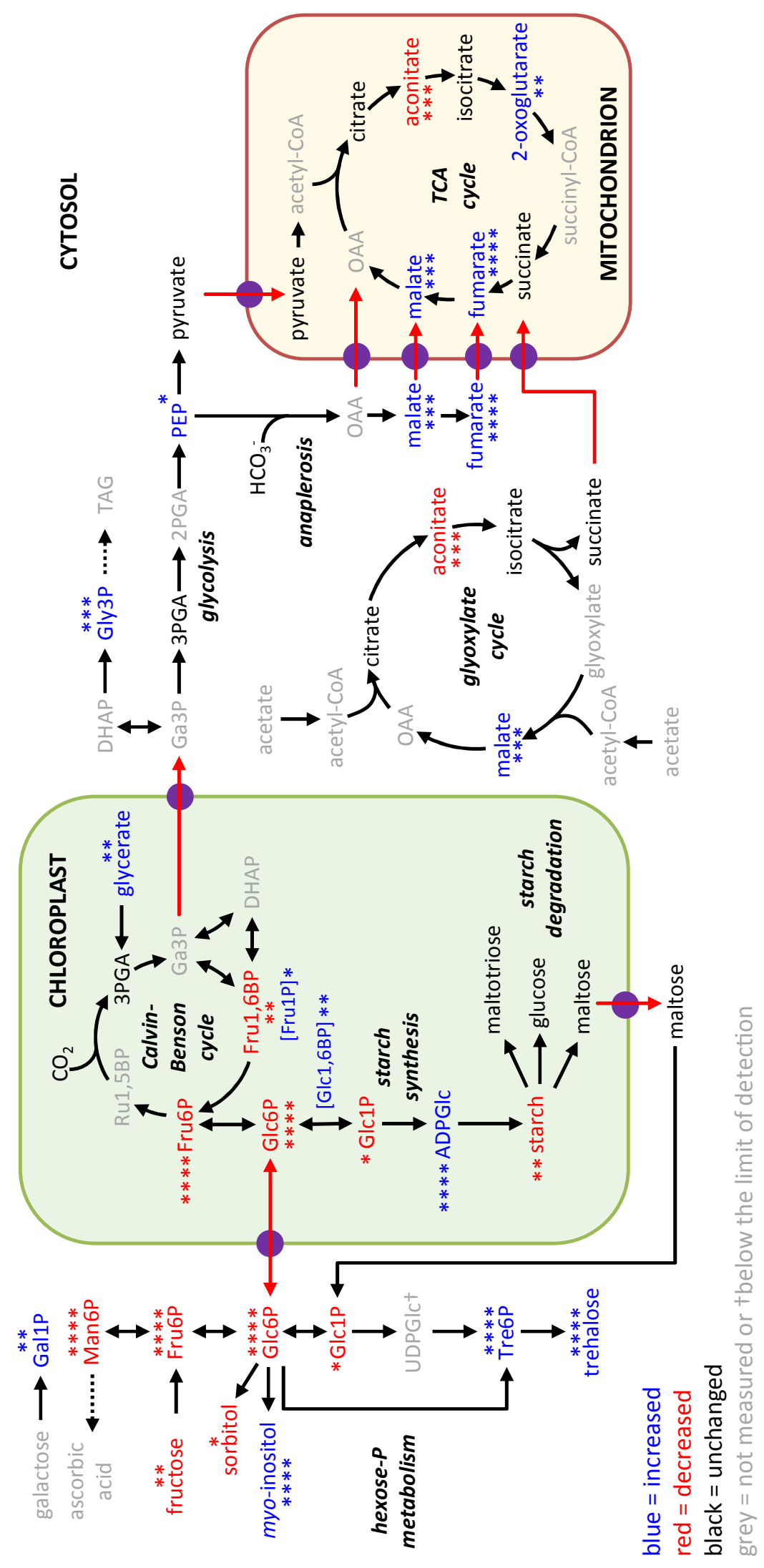


1052

1053

1054

1055

1056

1057

1058

1059

1060

1061

1062

1063

1064

1065

1066

1067

1068

1069

1070

1071

1072

\section{Figure 5. The overview of the selected metabolic pathways in gunSOS1 compared to} sigRep. The map was constructed based on the measured metabolites presented in Figures 3, 4, and Supplementary Figure 5. Metabolites increased in gunSOS1 compared to sigRep: fumaric acid (fumarate), oxoglutaric acid (2-OG), myo-Inositol, ADP-glucose (ADPGlc), galactose 1-phosphate (Gal1P), fructose 1-phosphate (Fru1P), alpha-D-Glucose 1,6bisphosphate (Glc1, 6BP), glycerol 3-phosphate (Gly3P), phosphoenolpyruvic acid (PEP), LMalic acid (malate). Metabolites decreased in gunSOS1 compared to sigRep: cis-Aconitic acid (aconitate), mannose 6-phosphate (Man6P), Glucose 6-phosphate (Glc6P), D-fructose (frictose), Fructose 6-phosphate (Fru6P), Glucose 1-phosphate (Glc1P), Fructose 1,6bisphosphate (Fru1, 6BP), and sorbitol. Metabolites that did not show significant difference in gunSOS1 relative to sigRep: D-Glucose (glucose), D-Maltose (maltose), maltotriose, citric acid (citrate), isocitric acid (isocitrate), pyruvic acid (pyruvate), succinic acid (succinate), 3phosphoglyceric acid (3PGA). The colour-coding: blue = increased, red = decreased, black =

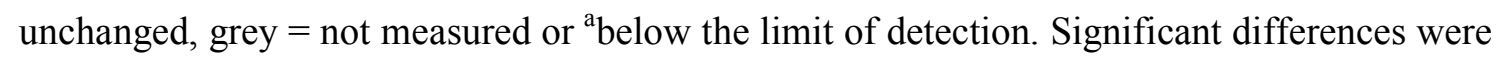
calculated using two-tailed Student's $t$-test. The maximal significant differences from any time point in light (Fig. 4 and 5) are indicated by asterisks, ${ }^{*} P<0.05, * * P<0.01, * * * P<$ 0.001 , and $* * * * P<0.0001$. The metabolic map was constructed based on the review by ${ }^{81}$ and ${ }^{82}$. 


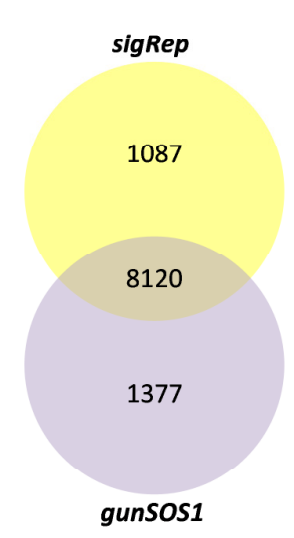

b

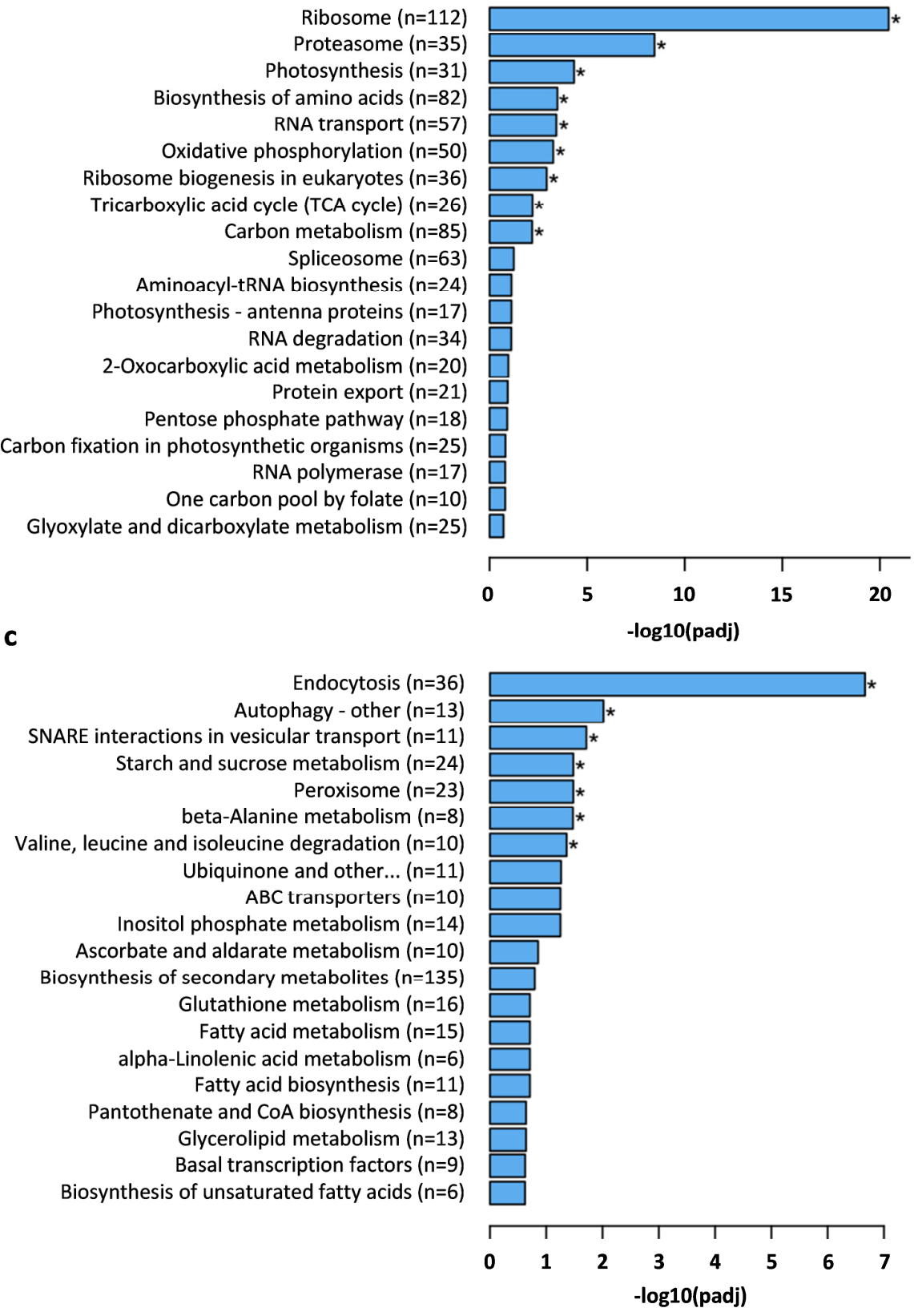

Figure 6. Transcriptome analysis based on RNA-seq. a Venn diagram showing number of genes expressed in gunSOS1 and sigRep (8120), or uniquely expressed in gunSOS1 (1377) or in sigRep (1087). b Pathway enrichment analysis (KEGG, http://www.kegg.jp/) based on the differentially expressed genes (RNA-seq); genes down-regulated in gunSOS1 compared to sigRep. c KEGG analysis showing genes up-regulated in gunSOS1 compared to sigRep. Effect on metabolic processes is sorted by the $p$ value. Asterisk indicates the core (significant) enrichment. 
a
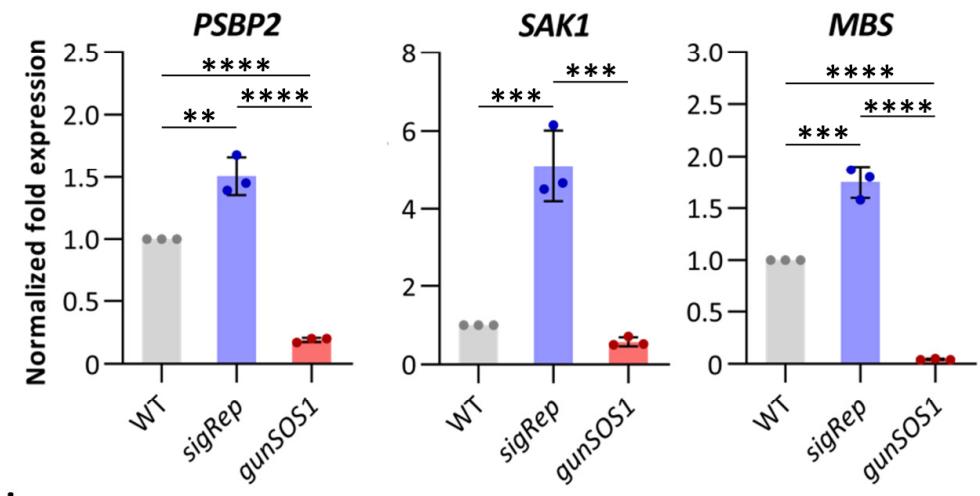

b
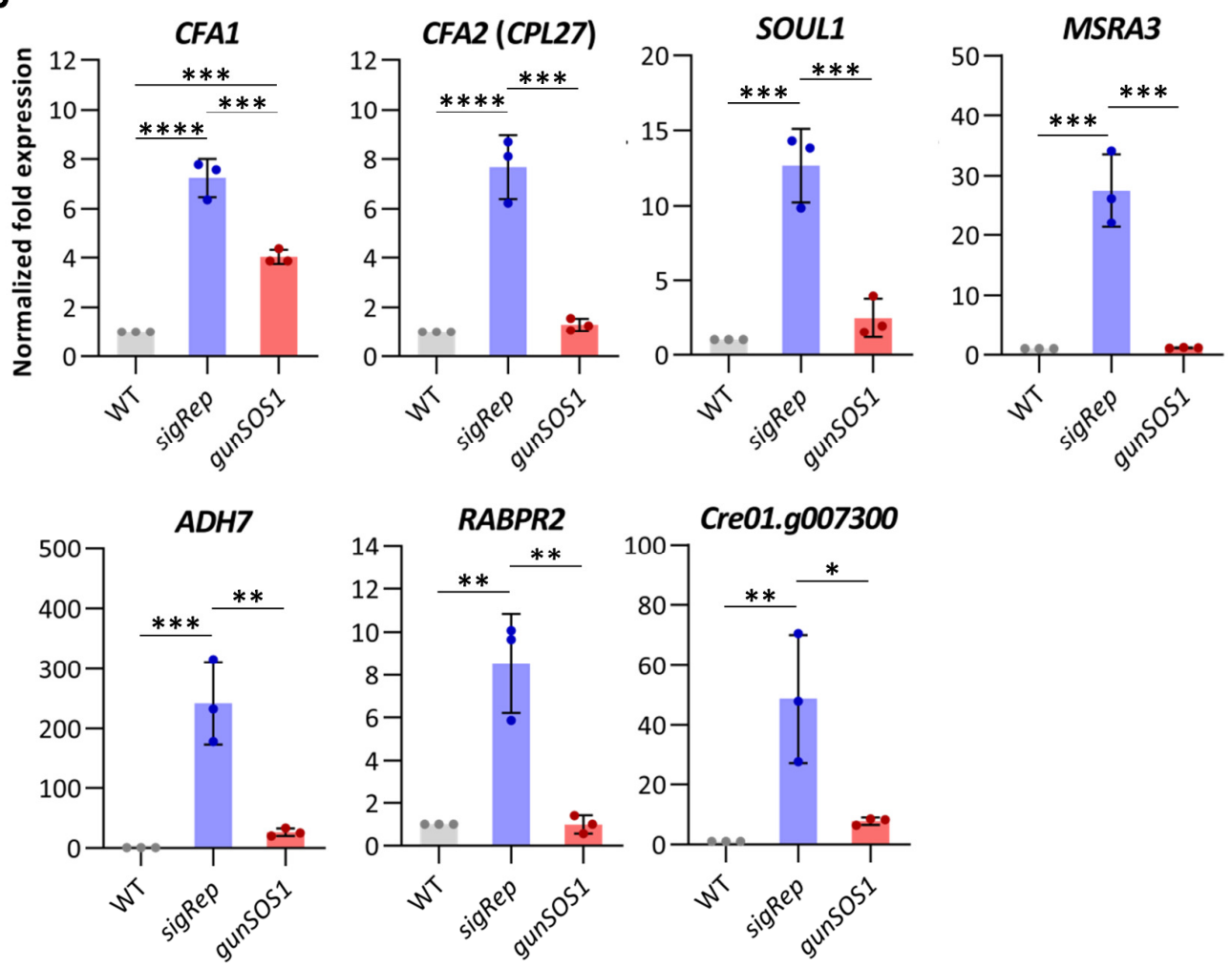

Figure 7. The qRT-PCR analysis of gene expression in gunSOS1 compared to sigRep. a

1084 Expression of genes encoding proteinaceous components previously associated with the 1085 chloroplast retrograde signalling involving ${ }^{1} \mathrm{O}_{2}, P S B P 2^{14}, S A K 1^{15}$, and $M B S^{16}$. b transcript 1086 levels of the selected genes showing attenuated expression in gunSOS1 compared to sigRep 1087 in the context of the SAK1-transcriptome ${ }^{15}$, genes description in the text. Experiments were performed in biological replications; results are presented as normalized fold expression $\left(2^{-}\right.$ $\Delta \Delta \mathrm{Ct}, \mathrm{WT}=1)$; the error bars represent calculated $\pm \mathrm{SD}$. Significant differences were calculated using one-way ANOVA, pair-wise comparison with the Tukey's post-hoc test (nonsignificant not shown), ${ }^{*} P<0.05, * * P<0.01, * * * P<0.001$, and $* * * * P<0.0001$. 
a

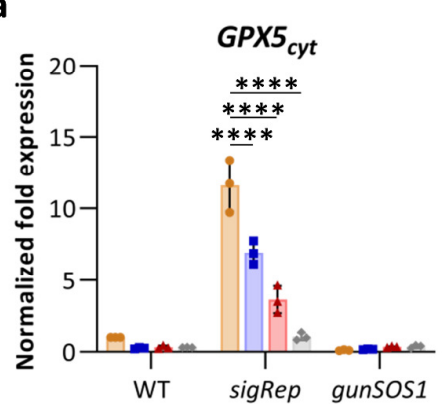

b

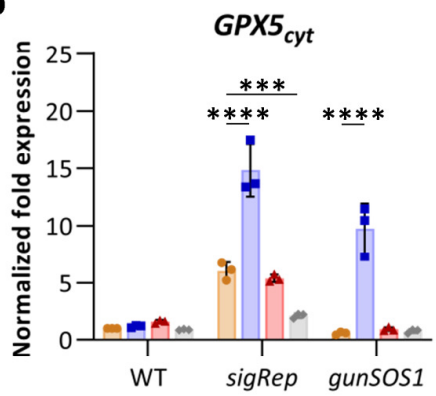

C

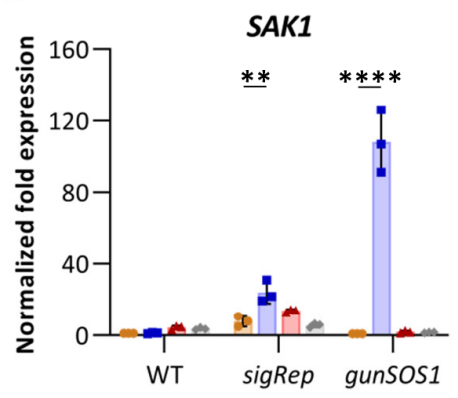

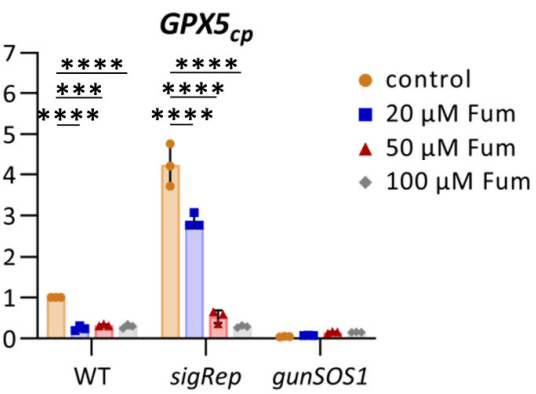

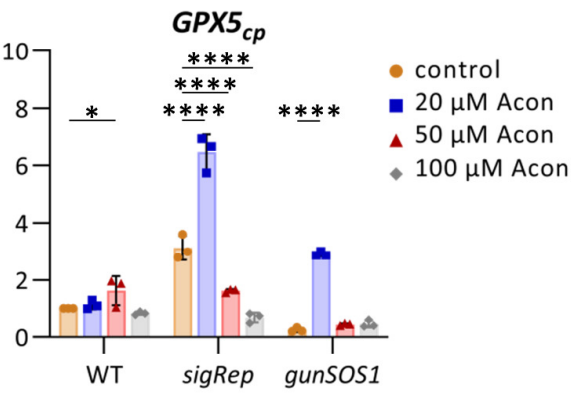

1092

1093

1094

1095

1096

1097

1098

1099

1100

1101

1102

1103

1104

1105

1106

1107

1108

1109

1110

1111

1112
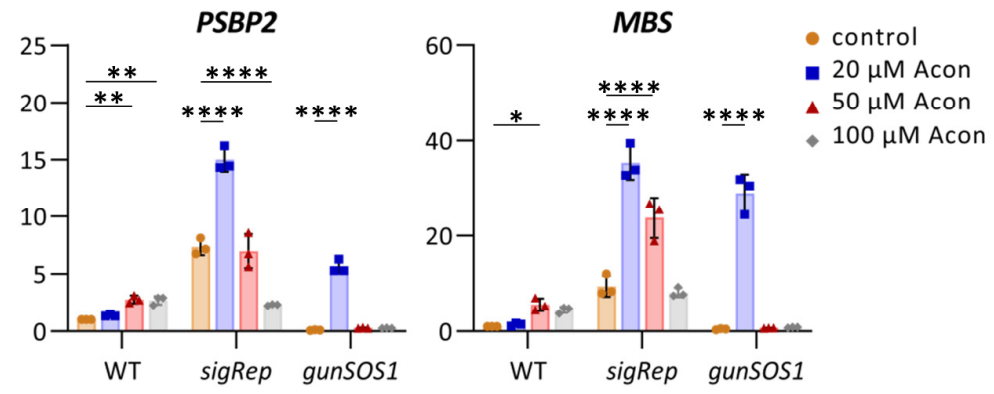


\section{Figure 8. Effect of selected metabolites applied exogenously on ${ }^{1} \mathrm{O}_{2}$-signalling inducing}

$1114 \boldsymbol{G P X 5}_{c y t}$ or $\boldsymbol{G P X 5}_{c p}$ expression. a Fumarate (Fum) significantly $(P<0.0001)$ decreased GPX5 expression in sigRep in concentration-dependent manner. Interaction between mutants and fumarate, as well as the mutant-dependent expression of GPX5 were also significant, $P<$ 11170.0001 and $P<0.001$, respectively. Fumarate had no effect on $G P X 5_{c y t}$ expression in 1118 gunSOS1 or WT, but attenuating effect was observed for $G P X 5_{c p}$ expression in WT $(P \leq$ 1119 0.0001). Detailed reports from statistical analyses are presented in Supplementary Tables 4 and 5. b Aconitate (Acon) at $20 \mu \mathrm{M}$ concentration rescues GPX5 expression in gunSOS1 and increases GPX5 expression in sigRep. Statistical analysis showed that $20 \mu \mathrm{M}$ aconitate significantly $(P \leq 0.0001)$ affect $G P X 5_{c y t}$ expression in gunSOS1 and sigRep, but $100 \mu \mathrm{M}$ concentration $(P=0.0001)$ decreased this expression in sigRep. An increase in $G P X 5_{c p}$ expression $(P<0.05)$ was also observed in WT upon treatment with $50 \mu \mathrm{M}$ aconitate (Supplementary Table 6 and 7). c Transcripts content for proteins necessary for the ${ }^{1} \mathrm{O}_{2}$ signalling, $S A K 1, P S B P 2$, and $M B S$ significantly $(P<0.01)$ increased upon treatment with 20 $\mu \mathrm{M}$ aconitate. An increase in PSBP2 and $M B S$ expression was also observed in WT treated with $50 \mu \mathrm{M}$ aconitate. Detailed statistical analyses are presented in Supplementary Tables 810. The qRT-PCR experiments were performed in biological replications $(n=3)$; results are presented as normalized fold expression $\left(2^{-\Delta \Delta \mathrm{Ct}}\right.$, untreated $\left.\mathrm{WT}=1\right)$; the error bars represent calculated \pm SD. Significant differences were calculated using two-way ANOVA with Dunnett's multiple comparison test and are indicated by asterisks (non-significant changes are not shown on the graph for clarity), $* P<0.05, * * P<0.01, * * * P<0.001$, and $* * * * P<$ 0.0001 . 
1137

1138

1139

1140

1141

1142

1143

1144

1145

1146

1147

1148

1149

1150

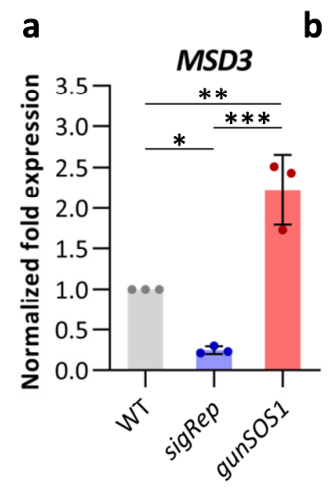

b

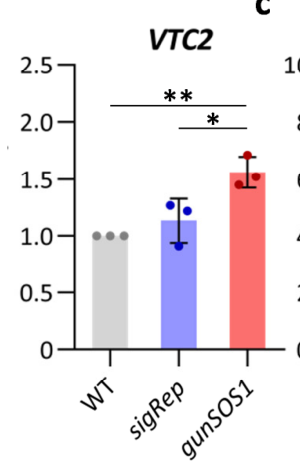

C
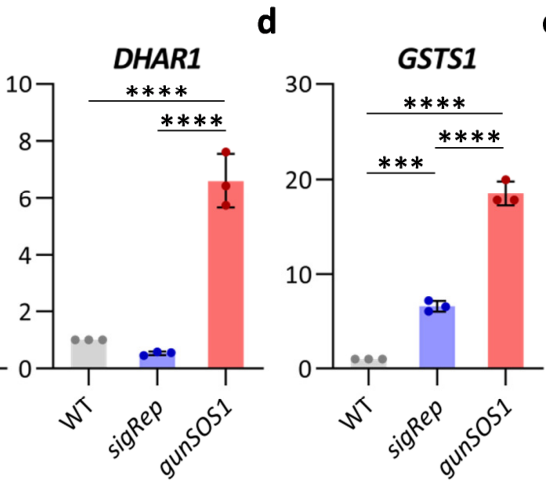

e

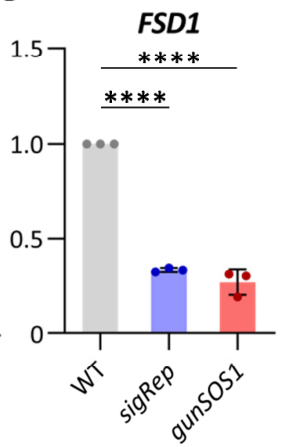

Figure 9. Expression of genes, which induction was previously associated with $\mathrm{H}_{2} \mathrm{O}_{2}$ or organic peroxides, but not with ${ }^{1} \mathbf{O}_{2}$. a Mn superoxide dismutase 3 (MSD3). b ASCORBATE PEROXIDASE 1 (APX1). c Mono-functional catalase (CAT1). d GDP-L-galactose Phosphorylase (VTC2). e monodehydroascorbate reductase (MDAR1). f dehydrosascorbate reductase (DHARl) g GLUTATHIONE REDUCTASE (GSHR1). h r-glutamylcysteine synthetase (GSH1). i Glutathione S-transferase (GSTS1). j Fe superoxide dismutase (FSD1). Experiments were performed in biological replications $(n=3)$; results are presented as normalized fold expression $\left(2^{-\Delta \Delta \mathrm{Ct}}, \mathrm{WT}=1\right)$; the error bars represent calculated $\pm \mathrm{SD}$. Significant differences were calculated using one-way ANOVA, pair-wise comparison with the Tukey's post-hoc test (non-significant not shown), ${ }^{*} P<0.05, * * P<0.01, * * * P<0.001$, and $* * * * P<0.0001$. 


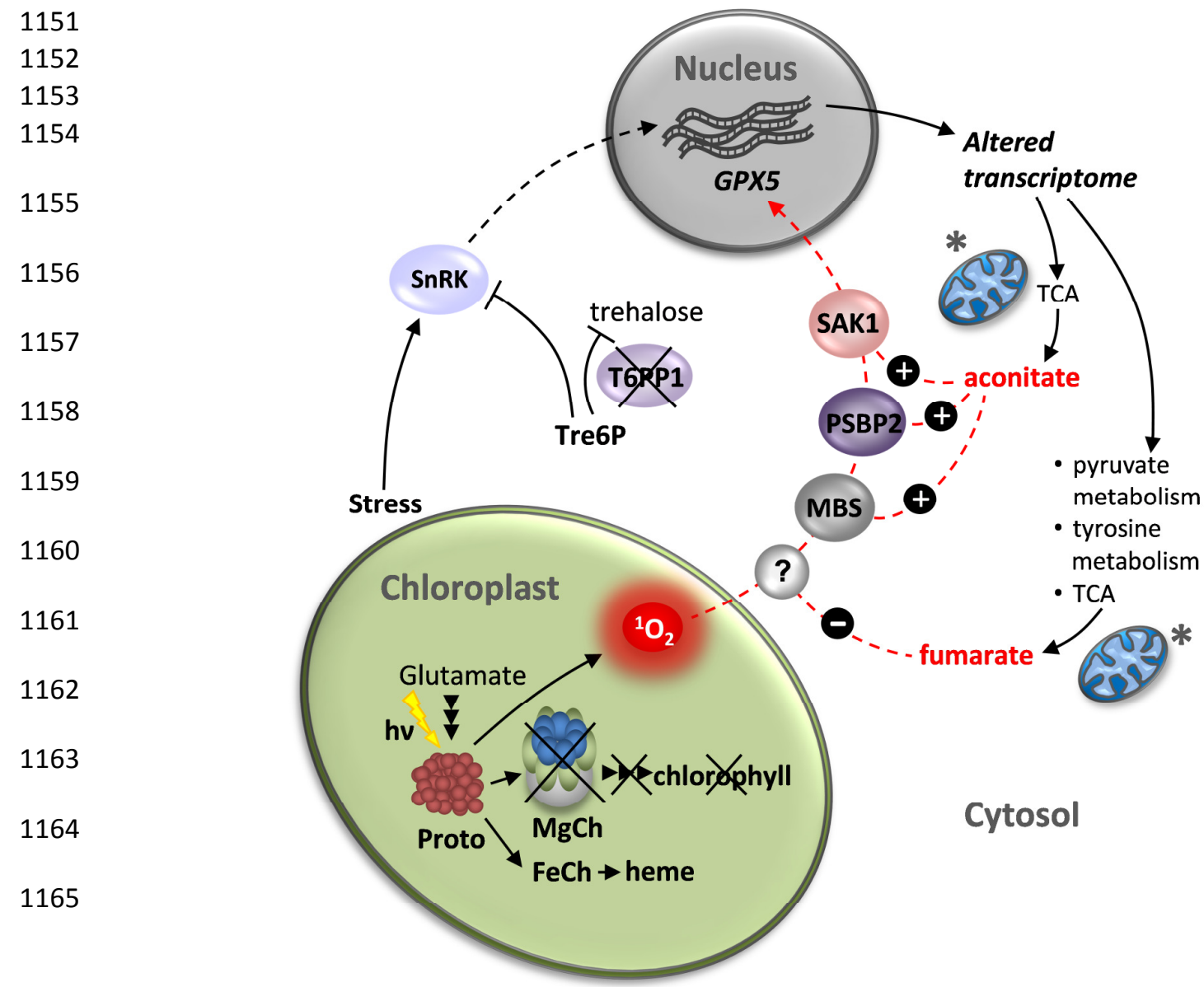

1166 Figure 10. Model of the chloroplast ${ }^{1} \mathrm{O}_{2}$-induced retrograde signalling depending on the 1167 metabolite content. Lack of functional $\mathrm{Mg}$-chelatase $(\mathrm{MgCh})$ leads to accumulation of 1168 protoporphyrin IX (Proto), which in light generates ${ }^{1} \mathrm{O}_{2}$. Lack of functional T6PP1 results in 1169 accumulation of trehalose 6-phosphate (Tre6P), which inhibits SnRK. Blocked SnRK 1170 signalling pathway leads to the inadequate transcriptomic response to stress and disturbed 1171 metabolites content. Fumarate accumulated due to the altered expression of genes involved in 1172 pyruvate metabolism, tyrosine metabolism and the TCA cycle. Accumulation of fumarate and 1173 disturbed TCA cycle lead to deficiency in aconitate. Fumarate attenuate ${ }^{1} \mathrm{O}_{2}$-signalling 1174 through yet unknown factor (marked with a question mark). The proteinaceous components, 1175 PSBP2, MBS, and SAK1, previously demonstrated to be required for ${ }^{1} \mathrm{O}_{2}$-signalling are 1176 shown. Aconitate is promoting expression of PSBP2, MBS, and SAK1 in gunSOS1 mutant. 1177 Mitochondria are marked by an asterisk. 